\title{
Constructing even radius tightly attached half-arc-transitive graphs of valency four
}

\author{
Yan-Quan Feng · Jin Ho Kwak • Chuixiang Zhou
}

Received: 16 November 2005 / Accepted: 8 February 2007 /

Published online: 17 April 2007

(C) Springer Science+Business Media, LLC 2007

\begin{abstract}
A finite graph $X$ is half-arc-transitive if its automorphism group is transitive on vertices and edges, but not on arcs. When $X$ is tetravalent, the automorphism group induces an orientation on the edges and a cycle of $X$ is called an alternating cycle if its consecutive edges in the cycle have opposite orientations. All alternating cycles of $X$ have the same length and half of this length is called the radius of $X$. The graph $X$ is said to be tightly attached if any two adjacent alternating cycles intersect in the same number of vertices equal to the radius of $X$. Marušič (J. Comb. Theory $B, 73,41-76,1998$ ) classified odd radius tightly attached tetravalent half-arctransitive graphs. In this paper, we classify the half-arc-transitive regular coverings of the complete bipartite graph $K_{4,4}$ whose covering transformation group is cyclic of prime-power order and whose fibre-preserving group contains a half-arc-transitive subgroup. As a result, two new infinite families of even radius tightly attached tetravalent half-arc-transitive graphs are constructed, introducing the first infinite families of tetravalent half-arc-transitive graphs of 2-power orders.
\end{abstract}

Keywords Graph · Half-arc-transitive · Tightly attached · Covering

Mathematics Subject Classification (2000) 05 C25 $\cdot 20 \mathrm{~B} 25$

Y.-Q. Feng $(\bowtie) \cdot$ C. Zhou

Department of Mathematics, Beijing Jiaotong University, Beijing 100044, People's Republic of China

e-mail: yqfeng@bjtu.edu.cn

C. Zhou

e-mail: zhouchuixiang@tom.com

J.H. Kwak

Department of Mathematics, Pohang University of Science and Technology, Pohang,

790-784 Korea

e-mail: jinkwak@postech.ac.kr 


\section{Introduction}

Throughout this paper, all graphs are assumed to be finite, connected, undirected and simple unless otherwise stated. For a graph $X$ we let $V(X), E(X), A(X)$ and $\operatorname{Aut}(X)$ denote the vertex, the edge, the arc set and the full automorphism group of $X$, respectively. A graph $X$ is said to be vertex-, edge-, or arc-transitive if $\operatorname{Aut}(X)$ acts transitively on $V(X), E(X)$ or $A(X)$, respectively. An edge- but not vertex-transitive graph with regular valency is called semisymmetric. Such a graph is necessarily bipartite. A graph is $\frac{1}{2}$-arc-transitive (or half-arc-transitive) if it is vertex- and edgebut not arc-transitive. More generally, by a $\frac{1}{2}$-arc-transitive action of a group $G$ on $X$ we shall mean a vertex- and edge- but not arc-transitive action of $G$ on $X$. In this case we say that the graph $X$ is $\left(G, \frac{1}{2}\right)$-arc-transitive.

The investigation of $\frac{1}{2}$-arc-transitive graphs was initiated by Tutte [43] who proved that a vertex- and edge-transitive graph with odd valency must be arc-transitive. In 1970, Bouwer [4] constructed the first infinite family of $\frac{1}{2}$-arc-transitive graphs and later more such graphs were constructed (see [1, 8, 15, 17, 30, 36, 42, 44]). Constructing and characterizing tetravalent $\frac{1}{2}$-arc-transitive graphs is currently an active topic in algebraic graph theory (see $[2,5,9,13,19,22,23,29,31-33,35$, $37-40,46,47])$. Apart from research in regular maps related to $\frac{1}{2}$-arc-transitive graphs (see [6] and [32]), and research regarding primitivity/imprimitivity of action for $\frac{1}{2}$-arc-transitive graphs (see [18] and [42]), there are two other directions research in $\frac{1}{2}$-arc-transitive graphs has taken. The first one deals with the study of tetravalent $\frac{1}{2}$-arc-transitive graphs with large order vertex stabilizers, initiated by Marušič and Nedela [34]. Our results have no immediate implications to this area of research because the $\frac{1}{2}$-arc-transitive graphs constructed in this paper have vertex stabilizers isomorphic to $\mathbb{Z}_{2}$. In this paper we explore the second of these two research directions and investigate the "attachment of alternating cycles" question for tetravalent $\frac{1}{2}$-arc-transitive graphs.

Let $X$ be a tetravalent $\left(G, \frac{1}{2}\right)$-arc-transitive graph with a subgroup $G$ of $\operatorname{Aut}(X)$. Then under the natural $G$-action on $V(X) \times V(X)$, the arc set $A(X)$ is partitioned into two $G$-orbits, say $A_{1}$ and $A_{2}$, which are paired with each other, that is, $A_{2}=\left\{(v, u) \mid(u, v) \in A_{1}\right\}$. Each of two corresponding oriented graphs $\left(V(X), A_{1}\right)$ and $\left(V(X), A_{2}\right)$ has out-valency and in-valency equal to 2 , and admits $G$ as a vertexand arc-transitive group of automorphisms. Moreover, each of them has $X$ as its underlying graph. Let $D_{G}(X)$ be one of these two oriented graphs, fixed from now on. For an arc $(u, v)$ in $D_{G}(X)$, we say that $u$ and $v$ are the tail and the head of the arc $(u, v)$, respectively. An even length cycle $C$ in $X$ is called a $G$-alternating cycle if the vertices of $C$ are alternatively the tail or the head in $D_{G}(X)$ of their two incident edges in $C$. It is proved [29, Proposition 2.4(i)] that all $G$-alternating cycles in $X$ have the same length and form a decomposition of the edge set of $X$. The half of this length is denoted by $r_{G}(X)$ and is called the $G$-radius of $X$. Two $G$-alternating cycles are said to be adjacent if they have at least one vertex in common. It is also shown [29, Proposition 2.6] that any two adjacent $G$-alternating cycles in $X$ intersect in the same number of vertices, called the $G$-attachment number of $X$ and denoted by $a_{G}(X)$. Furthermore, the $G$-attachment number $a_{G}(X)$ is a divisor of $2 r_{G}(X)$, twice the $G$-radius of $X$. If $X$ has only two $G$-alternating cycles then these cycles 
have the same set of vertices and $X$ is arc-transitive circulant, that is a Cayley graph on a cyclic group. These graphs were characterized in [29]. We say that $X$ is tightly $G$-attached if $a_{G}(X)=r_{G}(G)$ and loosely $G$-attached if $a_{G}(X)=1$. If two adjacent $G$-alternating cycles of $X$ intersect in a pair of antipodally opposite vertices then $X$ is said to be antipodally $G$-attached. If $X$ is $\frac{1}{2}$-arc-transitive, the terms an $\operatorname{Aut}(X)$ alternating cycle, $\operatorname{Aut}(X)$-radius, and $\operatorname{Aut}(X)$-attachment number are referred to as an alternating cycle of $X$, radius of $X$ and attachment number of $X$, respectively. Similarly, if $X$ is tightly $\operatorname{Aut}(X)$-attached, we say that $X$ is tightly attached.

Marušič and Praeger [37] proved that every finite connected tetravalent $\left(G, \frac{1}{2}\right)$-arctransitive graph is a cover of a smaller tetravalent $\left(G, \frac{1}{2}\right)$-arc-transitive graph which is tightly $G$-attached, loosely $G$-attached or antipodally $G$-attached. Thus, tightly, loosely and antipodally $G$-attached tetravalent graphs are basic ones for investigating tetravalent $\left(G, \frac{1}{2}\right)$-arc-transitive graphs and half-arc-transitive graphs. Wilson [45] demonstrated a variety of means for constructing $\frac{1}{2}$-arc-transitive graphs and completed the constructive characterization of tightly $G$-attached tetravalent $\left(G, \frac{1}{2}\right)$-arctransitive graphs considered in [29, 37]. Marušič [29] classified the tightly attached tetravalent $\frac{1}{2}$-arc-transitive graphs with odd radius, and the same graphs with even radius are definitely objects worth exploring. However, no even radius tightly attached tetravalent $\frac{1}{2}$-arc-transitive graph was given in these papers. In this paper, we construct two infinite families of tetravalent $\frac{1}{2}$-arc-transitive graphs that are of even radius and tightly attached. This may be a starting step for a possible classification of all even radius tightly attached tetravalent $\frac{1}{2}$-arc-transitive graphs of order a power of 2 .

\section{Main theorem}

Let $X$ be a connected graph. For $u, v \in V(X)$, we denote by $u v$ the edge incident to $u$ and $v$ in $X$, and by $N_{X}(u)$ the neighborhood of $u$ in $X$, that is, the set of vertices adjacent to $u$ in $X$. A graph $\widetilde{X}$ is called a covering of $X$ with a projection $p: \widetilde{X} \rightarrow X$, if $p$ is a surjection from $V(\tilde{X})$ to $V(X)$ such that $\left.p\right|_{N_{\tilde{X}}(\tilde{v})}: N_{\widetilde{X}}(\tilde{v}) \rightarrow N_{X}(v)$ is a bijection for any vertex $v \in V(X)$ and $\tilde{v} \in p^{-1}(v)$. The graph $\tilde{X}$ is called the covering graph and $X$ is the base graph. A covering $\widetilde{X}$ of $X$ with a projection $p$ is said to be regular (or $K$-covering) if there is a semiregular subgroup $K$ of the automorphism group $\operatorname{Aut}(\tilde{X})$ such that the orbits of $K$ on $V(\tilde{X})$ coincide with the sets $p^{-1}(v), v \in V(X)$. If $K$ is cyclic or elementary abelian then $\widetilde{X}$ is called a cyclic or an elementary abelian covering of $X$, and if $\widetilde{X}$ is connected $K$ becomes the covering transformation group. The fibre of an edge or a vertex is its preimage under $p$. An automorphism of $\widetilde{X}$ is said to be fibre-preserving if it maps a fibre to a fibre, while every covering transformation maps a fibre onto itself. All such fibre-preserving automorphisms form a group called the fibre-preserving group.

Covering techniques has been used as a powerful tool in topology and graph theory. The connected symmetric or semisymmetric coverings of a small graph have received considerable attention in recent years. For example, Malnič et al. [27] and Feng et al. [10] classified connected cubic semisymmetric or $s$-regular cyclic coverings of the bipartite graph $K_{3,3}$ for each $1 \leq s \leq 5$ when the fibre-preserving group 
contains an edge-, but not vertex-transitive or an arc-transitive subgroup, respectively. The $s$-regular cyclic or elementary abelian coverings of the hypercube $Q_{3}$ were classified in $[11,12]$ for each $1 \leq s \leq 5$ when the fibre-preserving group is arc-transitive. Furthermore, using a method developed in [25, 26], Malnič and Potočnik [24] classified connected vertex-transitive elementary abelian coverings of the Petersen graph when the fibre-preserving group is vertex-transitive. In order to obtain tetravalent $\frac{1}{2}$-arc-transitive graphs with special attachment property, Marušič and Waller [38] constructed infinitely many tetravalent $\frac{1}{2}$-arc-transitive graphs as cyclic coverings of $\frac{1}{2}$-arc-transitive graphs constructed in [23]. Note that the smallest $\frac{1}{2}$-arc-transitive graph has order 27 (see [46]) and the smallest valency of $\frac{1}{2}$-arc-transitive graphs is 4 . One may show that there is no $\frac{1}{2}$-arc-transitive cyclic coverings of a tetravalent graph with order less than 8 when the fibre-preserving group contains a $\frac{1}{2}$-arc-transitive subgroup. The purpose of this paper is to classify the connected $\frac{1}{2}$-arc-transitive regular coverings of the complete bipartite graph $K_{4,4}$ whose covering transformation group is cyclic of prime-power order and the fibre-preserving group contains a $\frac{1}{2}$-arc-transitive subgroup. As a result, two new infinite families of tetravalent $\frac{1}{2}$-arc-transitive graphs of 2-power orders are constructed, of which the smallest one has order $2^{7}$. All of these graphs have 2-power orders with girth 8 and force a lifted $\frac{1}{2}$-arc-transitive action to give rise to even radius tightly attached $\frac{1}{2}$-arc-transitive graphs. Also, it is easy to check that these graphs do not belong to any previously known families of $\frac{1}{2}$-arc-transitive graphs (note that the half-arc-transitive graphs constructed in [40, Theorem 3.1] have orders $2^{r}$ times an odd integer with $2 \leq r \leq 3$ (see [40, Lemma 3.5])).

To state the main theorem of this paper, we first introduce infinitely many tetravalent $\frac{1}{2}$-arc-transitive graphs of 2-power orders. Let $\mathbb{Z}_{n}$ be the cyclic group of order $n$, as well as the ring of integers modulo $n$. Denote by $\mathbb{Z}_{n}^{*}$ the multiplicative group of $\mathbb{Z}_{n}$ consisting of numbers coprime to $n$. Denote by $V\left(K_{4,4}\right)=\{\mathbf{a}, \mathbf{b}, \mathbf{c}, \mathbf{d}, \mathbf{u}, \mathbf{v}, \mathbf{w}, \mathbf{x}\}$ the vertex set of the bipartite graph $K_{4,4}$ as in Fig. 1 . Let $m \geq 4$ and let $k$ be an element of order 4 in $\mathbb{Z}_{2^{m}}^{*}$. The graphs $\mathcal{C K}\left(2^{m}, 1, k\right)$ and $\mathcal{C K}\left(2^{m}, 2, k\right)$ are defined to have the

Fig. 1 The graph $K_{4,4}$ with voltage assignment $\phi$

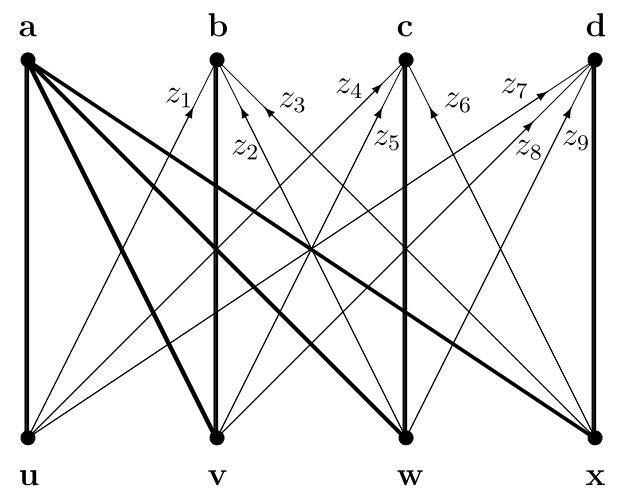


same vertex set $V\left(\mathcal{C K}\left(2^{m}, 1, k\right)\right)=V\left(\mathcal{C K}\left(2^{m}, 2, k\right)\right)=V\left(K_{4,4}\right) \times \mathbb{Z}_{2^{m}}$ and edge sets

$$
\begin{aligned}
E\left(\mathcal{C K}\left(2^{m}, 1, k\right)\right)= & \{(\mathbf{u}, x)(\mathbf{a}, x),(\mathbf{v}, x)(\mathbf{a}, x),(\mathbf{v}, x)(\mathbf{b}, x),(\mathbf{w}, x)(\mathbf{a}, x), \\
& (\mathbf{w}, x)(\mathbf{c}, x),(\mathbf{x}, x)(\mathbf{a}, x),(\mathbf{x}, x)(\mathbf{d}, x),(\mathbf{u}, x)(\mathbf{b}, x+2 k), \\
& (\mathbf{u}, x)\left(\mathbf{c}, x+k+2^{m-1}\right),(\mathbf{u}, x)(\mathbf{d}, x+k), \\
& (\mathbf{v}, x)\left(\mathbf{c}, x+2^{m-1}-1\right),(\mathbf{v}, x)(\mathbf{d}, x+1), \\
& (\mathbf{w}, x)\left(\mathbf{b}, x+k+2^{m-1}+1\right),(\mathbf{w}, x)(\mathbf{d}, x+k+1), \\
& \left.(\mathbf{x}, x)\left(\mathbf{b}, x+k+2^{m-1}-1\right),(\mathbf{x}, x)(\mathbf{c}, x+k-1) \mid x \in \mathbb{Z}_{2^{m}}\right\},
\end{aligned}
$$

$$
\begin{aligned}
E\left(\mathcal{C K}\left(2^{m}, 2, k\right)\right)= & \{(\mathbf{u}, x)(\mathbf{a}, x),(\mathbf{v}, x)(\mathbf{a}, x),(\mathbf{v}, x)(\mathbf{b}, x),(\mathbf{w}, x)(\mathbf{a}, x), \\
& (\mathbf{w}, x)(\mathbf{c}, x),(\mathbf{x}, x)(\mathbf{a}, x),(\mathbf{x}, x)(\mathbf{d}, x), \\
& (\mathbf{u}, x)\left(\mathbf{b}, x+2 k+2^{m-1}\right),(\mathbf{u}, x)\left(\mathbf{c}, x+k+2^{m-1}\right), \\
& (\mathbf{u}, x)(\mathbf{d}, x+k), \quad(\mathbf{v}, x)(\mathbf{c}, x-1), \\
& (\mathbf{v}, x)(\mathbf{d}, x+1), \quad(\mathbf{w}, x)(\mathbf{b}, x+k+1), \\
& (\mathbf{w}, x)\left(\mathbf{d}, x+k+2^{m-1}+1\right), \\
& \left.(\mathbf{x}, x)\left(\mathbf{b}, x+k+2^{m-1}-1\right),(\mathbf{x}, x)(\mathbf{c}, x+k-1) \mid x \in \mathbb{Z}_{2^{m}}\right\},
\end{aligned}
$$

respectively. The notation $\mathcal{C K}$ means a cyclic covering. It is easy to see that $\mathcal{C K}\left(2^{m}, 1, k\right)$ and $\mathcal{C K}\left(2^{m}, 2, k\right)$ are bipartite graphs. In fact, both are cyclic coverings of $K_{4,4}$. Since $\mathbb{Z}_{2^{m}}^{*} \cong \mathbb{Z}_{2} \times \mathbb{Z}_{2^{m-2}}$ for each $m \geq 4$, there are exactly 4 elements of order 4 in $\mathbb{Z}_{2^{m}}^{*}$, that is, $2^{m-2} \pm 1$ and $2^{m-2}+2^{m-1} \pm 1$. It will be shown that the graphs $\mathcal{C K}\left(2^{m}, i, 2^{m-2}+1\right), \mathcal{C K}\left(2^{m}, i, 2^{m-2}-1\right), \mathcal{C K}\left(2^{m}, i, 2^{m-2}+2^{m-1}+1\right)$ and $\mathcal{C K}\left(2^{m}, i, 2^{m-2}+2^{m-1}-1\right)$ are isomorphic one another for each $i=1,2$ in Lemma 4.1. Thus, the graphs $\mathcal{C K}\left(2^{m}, 1, k\right)$ and $\mathcal{C K}\left(2^{m}, 2, k\right)$ are independent of the choice of the element $k$ of order 4 and we simply denote them by $\mathcal{C K}\left(2^{m}, 1\right)$ and $\mathcal{C K}\left(2^{m}, 2\right)$, respectively. The main result of this paper is the following theorem.

Theorem 2.1 Let $\widetilde{X}$ be a connected regular covering of the complete bipartite graph $K_{4,4}$ whose fibre-preserving group contains a $\frac{1}{2}$-arc-transitive subgroup. If the covering transformation group is a cyclic group $\mathbb{Z}_{p^{m}}$ of order $p^{m}$ for a prime $p$ then $\widetilde{X}$ is $\frac{1}{2}$-arc-transitive if and only if $p=2, m \geq 4$ and $\widetilde{X} \cong \mathcal{C K}\left(2^{m}, 1\right)$ or $\mathcal{C K}\left(2^{m}, 2\right)$. Furthermore, all of these graphs are tightly attached with radius $2^{m}$.

The smallest graphs in the two infinite families of half-arc-transitive graphs given in Theorem 2.1 are $\mathcal{C K}(16,1)$ and $\mathcal{C K}(16,2)$, of which the first is depicted in Fig. 2. These two smallest graphs have 128 vertices, and we do not know any example of even radius tightly attached half-arc-transitive graphs of valency 4 with order less than 128 .

To end the section, we lay out the strategy of the proof of Theorem 2.1. Let $\widetilde{X}$ be a connected regular covering of the graph $K_{4,4}$ satisfying the hypotheses in Theorem 2.1. Then a minimal $\frac{1}{2}$-arc-transitive subgroup of $\operatorname{Aut}\left(K_{4,4}\right)$ lifts (or, equivalently the fibre-preserving group contains a $\frac{1}{2}$-arc-transitive subgroup). There are only three minimal $\frac{1}{2}$-arc-transitive groups of $\operatorname{Aut}\left(K_{4,4}\right)$ up to conjugacy, say $G_{1}, G_{2}$ or $G_{3}$. In Sect. 5, we deal with the three cases: $G_{1}, G_{2}$ or $G_{3}$ lifts, respectively. In particular, $\widetilde{X}$ cannot be $\frac{1}{2}$-arc-transitive when $G_{2}$ or $G_{3}$ lifts (Lemmas 5.3 and 5.4) and infinitely 


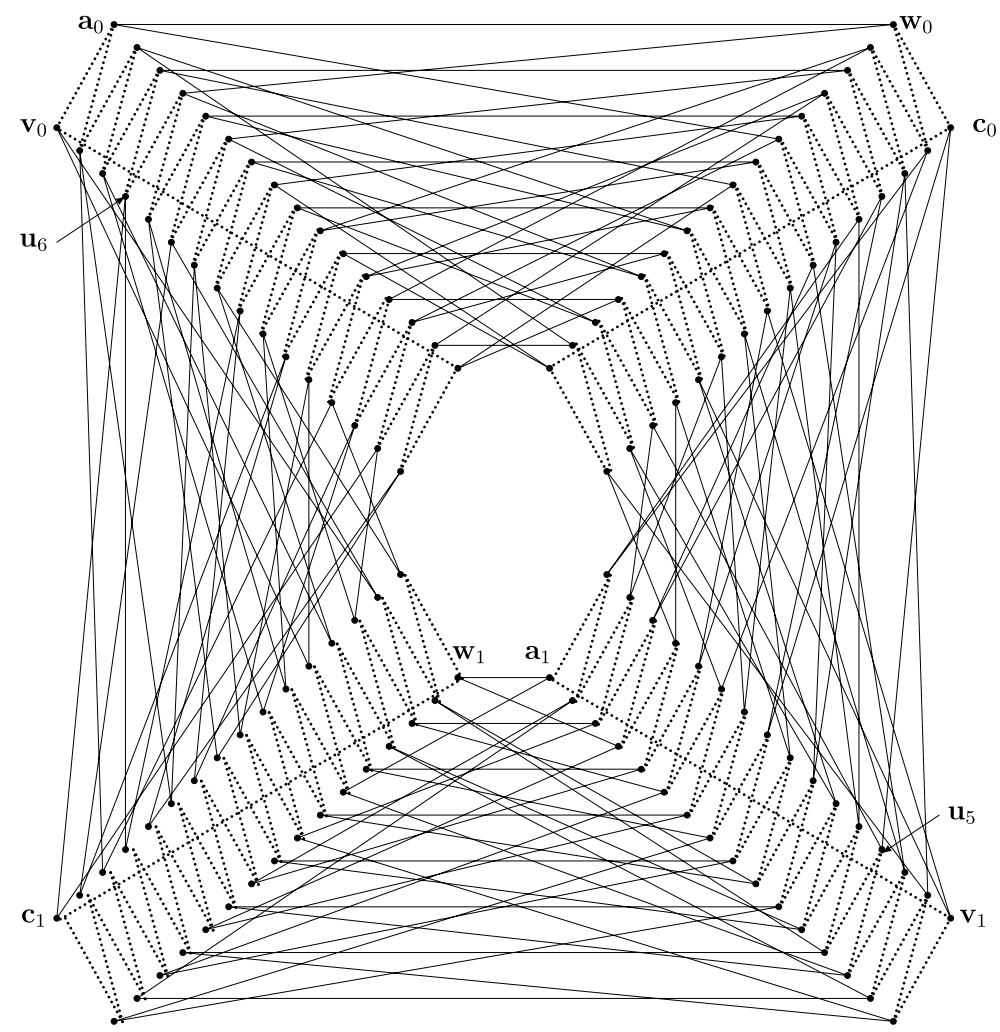

Fig. 2 The graph $\mathcal{C K}(16,1,3)=\mathcal{C K}(16,1)$

many tetravalent $\frac{1}{2}$-arc-transitive graphs are constructed when $G_{1}$ lifts (Lemmas 5.1 and 5.2), of which the pairwise non-isomorphic ones are given in Sect. 4.

\section{Voltage graphs and lifting problems}

Let $X$ be a graph and $K$ a finite group. By $a^{-1}$ we mean the reverse arc to an arc $a$. A voltage assignment (or, $K$-voltage assignment) of $X$ is a function $\phi: A(X) \rightarrow K$ with the property that $\phi\left(a^{-1}\right)=\phi(a)^{-1}$ for each arc $a \in A(X)$. The values of $\phi$ are called voltages, and $K$ is the voltage group. The graph $X \times_{\phi} K$ derived from a voltage assignment $\phi: A(X) \rightarrow K$ has vertex set $V(X) \times K$ and edge set $E(X) \times K$, so that an edge $(e, g)$ of $X \times{ }_{\phi} K$ joins a vertex $(u, g)$ to $(v, \phi(a) g)$ for $a=(u, v) \in A(X)$ and $g \in K$, where $e=u v$.

Clearly, the derived graph $X \times_{\phi} K$ is a covering of $X$ with the first coordinate projection $p: X \times_{\phi} K \rightarrow X$, which is called the natural projection. By defining $\left(u, g^{\prime}\right)^{g}:=\left(u, g^{\prime} g\right)$ for any $g \in K$ and $\left(u, g^{\prime}\right) \in V\left(X \times_{\phi} K\right), K$ becomes a subgroup of $\operatorname{Aut}\left(X \times_{\phi} K\right)$ which acts semiregularly on $V\left(X \times_{\phi} K\right)$. Therefore, $X \times_{\phi} K$ can be viewed as a $K$-covering. For each $u \in V(X)$ and $u v \in E(X)$, the vertex set $\{(u, g) \mid g \in K\}$ is the fibre of $u$ and the edge set $\{(u, g)(v, \phi(a) g) \mid g \in K\}$ is the 
fibre of $u v$, where $a=(u, v)$. Conversely, each regular covering $\widetilde{X}$ of $X$ with a covering transformation group $K$ can be derived from a $K$-voltage assignment. Given a spanning tree $T$ of the graph $X$, a voltage assignment $\phi$ is said to be $T$-reduced if the voltages on the tree arcs are the identity. Gross and Tucker [14] showed that every regular covering $\widetilde{X}$ of a graph $X$ can be derived from a $T$-reduced voltage assignment $\phi$ with respect to an arbitrary fixed spanning tree $T$ of $X$. It is clear that if $\phi$ is reduced, the derived graph $X \times_{\phi} K$ is connected if and only if the voltages on the cotree arcs generate the voltage group $K$.

Let $\widetilde{X}$ be a $K$-covering of $X$ with a projection $p$. If $\alpha \in \operatorname{Aut}(X)$ and $\widetilde{\alpha} \in \operatorname{Aut}(\tilde{X})$ satisfy $\widetilde{\alpha} p=p \alpha$, we call $\widetilde{\alpha}$ a lift of $\alpha$, and $\alpha$ the projection of $\widetilde{\alpha}$ (for the purpose of this paper, all mappings are composed from left to right). Concepts such as a lift of a subgroup of $\operatorname{Aut}(X)$ and the projection of a subgroup of $\operatorname{Aut}(\tilde{X})$ are self-explanatory. The lifts and the projections of such subgroups are of course subgroups in $\operatorname{Aut}(\widetilde{X})$ and $\operatorname{Aut}(X)$, respectively. In particular, if the covering graph $\widetilde{X}$ is connected, then the covering transformation group $K$ is the lift of the trivial group, that is, $K=\{\widetilde{\alpha} \in \operatorname{Aut}(\widetilde{X}): p=\widetilde{\alpha} p\}$. Clearly, all possible lifts of automorphisms of $X$ form a group and one may show that this group is exactly the fibre-preserving group.

Let $X \times_{\phi} K \rightarrow X$ be a connected $K$-covering derived from a $T$-reduced voltage assignment $\phi$. The problem whether an automorphism $\alpha$ of $X$ lifts or not can be grasped in terms of voltages as follows. Observe that a voltage assignment on arcs extends to a voltage assignment on walks in a natural way. Given $\alpha \in \operatorname{Aut}(X)$, we define a function $\bar{\alpha}$ from the set of voltages of fundamental closed walks based at a fixed vertex $v \in V(X)$ to the voltage group $K$ by

$$
(\phi(C))^{\bar{\alpha}}=\phi\left(C^{\alpha}\right)
$$

where $C$ runs over all fundamental closed walks at $v$, and $\phi(C)$ and $\phi\left(C^{\alpha}\right)$ are the voltages of $C$ and $C^{\alpha}$, respectively. Note that if $K$ is abelian, $\bar{\alpha}$ does not depend on the choice of the base vertex, and the fundamental closed walks at $v$ can be substituted by the fundamental cycles generated by the cotree arcs of $X$.

The next proposition is a special case of [20, Theorem 4.2].

Proposition 3.1 Let $X \times_{\phi} K \rightarrow X$ be a connected $K$-covering derived from $a T$-reduced voltage assignment $\phi$. Then an automorphism $\alpha$ of $X$ lifts if and only if $\bar{\alpha}$ extends to an automorphism of $K$.

For more results on the lifts of automorphisms of $X$, we refer the reader to $[7,21,28]$.

Two coverings $\widetilde{X}_{1}$ and $\widetilde{X}_{2}$ of $X$ with projections $p_{1}$ and $p_{2}$ respectively, are said to be equivalent if there exists a graph isomorphism $\widetilde{\alpha}: \widetilde{X}_{1} \rightarrow \widetilde{X}_{2}$ such that $\widetilde{\alpha} p_{2}=p_{1}$. We quote the following proposition.

Proposition $3.2[16,41]$ Two connected regular coverings $X \times_{\phi} K$ and $X \times_{\psi} K$, where $\phi$ and $\psi$ are $T$-reduced, are equivalent if and only if there exists an automorphism $\sigma \in \operatorname{Aut}(K)$ such that $\phi(u, v)^{\sigma}=\psi(u, v)$ for any cotree arc $(u, v)$ of $X$. 


\section{Isomorphism types of constructed graphs}

Let $m \geq 4$ and let $k$ be an element of order 4 in $\mathbb{Z}_{2^{m}}^{*}$. Then $k=2^{m-2} \pm 1$ or $2^{m-2}+2^{m-1} \pm 1$. In this section we shall prove that the graphs $\mathcal{C K}\left(2^{m}, i, 2^{m-2}+1\right)$ $\mathcal{C K}\left(2^{m}, i, 2^{m-2}-1\right), \mathcal{C K}\left(2^{m}, i, 2^{m-2}+2^{m-1}+1\right)$ and $\mathcal{C K}\left(2^{m}, i, 2^{m-2}+2^{m-1}-1\right)$ (defined in Sect. 2) are pairwise isomorphic for each $i=1,2$.

Lemma 4.1 Let $m \geq 4$ and $i=1$ or 2 . Then $\mathcal{C K}\left(2^{m}, i, 2^{m-2}+1\right), \mathcal{C K}\left(2^{m}, i\right.$, $\left.2^{m-2}-1\right), \mathcal{C K}\left(2^{m}, i, 2^{m-2}+2^{m-1}+1\right)$ and $\mathcal{C K}\left(2^{m}, i, 2^{m-2}+2^{m-1}-1\right)$ are pairwise isomorphic.

Proof Recall that all graphs $\mathcal{C K}\left(2^{m}, i, 2^{m-2}+1\right), \mathcal{C K}\left(2^{m}, i, 2^{m-2}-1\right), \mathcal{C K}\left(2^{m}, i\right.$, $\left.2^{m-2}+2^{m-1}+1\right)$ and $\mathcal{C K}\left(2^{m}, i, 2^{m-2}+2^{m-1}-1\right)$ have the same vertex set $V\left(K_{4,4}\right) \times \mathbb{Z}_{2^{m}}$. For small integers $m$, one can find an isomorphism from $\mathcal{C K}\left(2^{m}, 1\right.$, $\left.2^{m-2}+1\right)$ to $\mathcal{C K}\left(2^{m}, 1,2^{m-2}-1\right)$ by direct observation. From such isomorphisms, one can derive a map $\alpha: \mathcal{C K}\left(2^{m}, 1,2^{m-2}+1\right) \mapsto \mathcal{C K}\left(2^{m}, 1,2^{m-2}-1\right)$ for any integer $m \geq 4$ as follows:

$$
\begin{aligned}
& (\mathbf{a}, x) \mapsto\left(\mathbf{a},\left(2^{m-2}+2^{m-1}+1\right) x\right) \\
& (\mathbf{b}, x) \mapsto\left(\mathbf{b},\left(2^{m-2}+2^{m-1}+1\right) x+2^{m-2}+2^{m-1}-2\right) \\
& (\mathbf{c}, x) \mapsto\left(\mathbf{c},\left(2^{m-2}+2^{m-1}+1\right) x+2^{m-1}-1\right) \\
& (\mathbf{d}, x) \mapsto\left(\mathbf{d},\left(2^{m-2}+2^{m-1}+1\right) x+2^{m-2}-1\right) \\
& (\mathbf{u}, x) \mapsto\left(\mathbf{w},\left(2^{m-2}+2^{m-1}+1\right) x\right) \\
& (\mathbf{w}, x) \mapsto\left(\mathbf{v},\left(2^{m-2}+2^{m-1}+1\right) x\right) \\
& (\mathbf{v}, x) \mapsto\left(\mathbf{x},\left(2^{m-2}+2^{m-1}+1\right) x\right) \\
& (\mathbf{x}, x) \mapsto\left(\mathbf{u},\left(2^{m-2}+2^{m-1}+1\right) x\right)
\end{aligned}
$$

where $x \in \mathbb{Z}_{2^{m}}$. By the definitions of $\mathcal{C K}\left(2^{m}, 1,2^{m-2}+1\right)$ and $\mathcal{C K}\left(2^{m}, 1,2^{m-2}-1\right)$,

$$
\begin{aligned}
& N_{\mathcal{C K}\left(2^{m}, 1,2^{m-2}+1\right)}((\mathbf{u}, x)) \\
& \quad=\left\{(\mathbf{a}, x),\left(\mathbf{b}, x+2^{m-1}+2\right),\left(\mathbf{c}, x+2^{m-2}+2^{m-1}+1\right),\left(\mathbf{d}, x+2^{m-2}+1\right)\right\}
\end{aligned}
$$

and

$$
\begin{aligned}
N_{\mathcal{C K}\left(2^{m}, 1,2^{m-2}-1\right)}\left((\mathbf{u}, x)^{\alpha}\right)=N_{\mathcal{C K}\left(2^{m}, 1,2^{m-2}-1\right)}\left(\left(\mathbf{w},\left(2^{m-2}+2^{m-1}+1\right) x\right)\right) \\
=\left\{\left(\mathbf{a},\left(2^{m-2}+2^{m-1}+1\right) x\right),\left(\mathbf{b},\left(2^{m-2}+2^{m-1}+1\right) x+2^{m-2}+2^{m-1}\right),\right. \\
\left.\quad\left(\mathbf{c},\left(2^{m-2}+2^{m-1}+1\right) x\right),\left(\mathbf{d},\left(2^{m-2}+2^{m-1}+1\right) x+2^{m-2}\right)\right\}
\end{aligned}
$$

where $N_{\mathcal{C K}\left(2^{m}, 1,2^{m-2}+1\right)}((\mathbf{u}, x))$ and $N_{\mathcal{C K}\left(2^{m}, 1,2^{m-2}-1\right)}\left((\mathbf{u}, x)^{\alpha}\right)$ are the neighborhoods of $(\mathbf{u}, x)$ and $(\mathbf{u}, x)^{\alpha}$ in $\mathcal{C K}\left(2^{m}, 1,2^{m-2}+1\right)$ and $\mathcal{C K}\left(2^{m}, 1,2^{m-2}-1\right)$, respectively.

Now, one can easily show that

$$
\left[N_{\mathcal{C K}\left(2^{m}, 1,2^{m-2}+1\right)}((\mathbf{u}, x))\right]^{\alpha}=N_{\mathcal{C K}\left(2^{m}, 1,2^{m-2}-1\right)}\left((\mathbf{u}, x)^{\alpha}\right) .
$$


Similarly, one can show that

$$
\left[N_{\mathcal{C K}\left(2^{m}, 1,2^{m-2}+1\right)}((\mathbf{z}, x))\right]^{\alpha}=N_{\mathcal{C K}\left(2^{m}, 1,2^{m-2}-1\right)}\left((\mathbf{z}, x)^{\alpha}\right) .
$$

for $\mathbf{z}=\mathbf{v}, \mathbf{w}$ or $\mathbf{x}$ and $x \in \mathbb{Z}_{2^{m}}$. This implies that $\alpha$ is an isomorphism from $\mathcal{C K}\left(2^{m}, 1,2^{m-2}+1\right)$ to $\mathcal{C K}\left(2^{m}, 1,2^{m-2}-1\right)$ because these two graphs are bipartite, as required.

Set $t_{1}=2^{m-2}+1, t_{2}=2^{m-2}-1, t_{3}=2^{m-1}+2^{m-2}+1$ and $t_{4}=2^{m-1}+2^{m-2}-1$. With a similar argument as above, one can prove that the following two maps

$$
\begin{array}{lll}
(\mathbf{a}, x) \mapsto\left(\mathbf{a}, t_{1} x\right) & (\mathbf{a}, x) \mapsto\left(\mathbf{a}, t_{2} x\right) \\
(\mathbf{b}, x) \mapsto\left(\mathbf{b}, t_{1} x+2^{m-2}\right) & & (\mathbf{b}, x) \mapsto\left(\mathbf{b}, t_{2} x+2^{m-2}-2\right) \\
(\mathbf{c}, x) \mapsto\left(\mathbf{d}, t_{1} x+t_{1}+2^{m-1}\right) & & (\mathbf{c}, x) \mapsto\left(\mathbf{d}, t_{2} x+t_{2}+2^{m-1}\right) \\
(\mathbf{d}, x) \mapsto\left(\mathbf{c}, t_{1} x+2^{m-1}-1\right) & \text { and } & (\mathbf{d}, x) \mapsto\left(\mathbf{c}, t_{2} x+2^{m-1}-1\right) \\
(\mathbf{u}, x) \mapsto\left(\mathbf{w}, t_{1} x\right) & & (\mathbf{u}, x) \mapsto\left(\mathbf{w}, t_{2} x\right) \\
(\mathbf{v}, x) \mapsto\left(\mathbf{x}, t_{1} x\right) & (\mathbf{v}, x) \mapsto\left(\mathbf{x}, t_{2} x\right) \\
(\mathbf{w}, x) \mapsto\left(\mathbf{u}, t_{1} x\right) & (\mathbf{w}, x) \mapsto\left(\mathbf{u}, t_{2} x\right) \\
(\mathbf{x}, x) \mapsto\left(\mathbf{v}, t_{1} x\right) & (\mathbf{x}, x) \mapsto\left(\mathbf{v}, t_{2} x\right)
\end{array}
$$

are actually isomorphisms from $\mathcal{C K}\left(2^{m}, 1,2^{m-2}+1\right)$ to $\mathcal{C K}\left(2^{m}, 1,2^{m-2}+2^{m-1}+1\right)$ and from $\mathcal{C K}\left(2^{m}, 1,2^{m-2}-1\right)$ to $\mathcal{C K}\left(2^{m}, 1,2^{m-2}+2^{m-1}-1\right)$, respectively, where $x \in \mathbb{Z}_{2^{m}}$. And the following three maps

$$
\begin{array}{lll}
(\mathbf{a}, x) \mapsto(\mathbf{a},-x) & (\mathbf{a}, x) \mapsto\left(\mathbf{a}, t_{3} x\right) & (\mathbf{a}, x) \mapsto\left(\mathbf{a}, t_{4} x\right) \\
(\mathbf{b}, x) \mapsto(\mathbf{b},-x) & (\mathbf{b}, x) \mapsto\left(\mathbf{b}, t_{3} x+2^{m-2}\right) & (\mathbf{b}, x) \mapsto\left(\mathbf{b}, t_{4} x+2^{m-2}-2\right) \\
(\mathbf{c}, x) \mapsto(\mathbf{d},-x) & (\mathbf{c}, x) \mapsto\left(\mathbf{d}, t_{3} x+t_{3}\right) & (\mathbf{c}, x) \mapsto\left(\mathbf{d}, t_{4} x+t_{4}\right) \\
(\mathbf{d}, x) \mapsto(\mathbf{c},-x) & (\mathbf{d}, x) \mapsto\left(\mathbf{c}, t_{3} x-1\right) & (\mathbf{d}, x) \mapsto\left(\mathbf{c}, t_{4} x-1\right) \\
(\mathbf{u}, x) \mapsto(\mathbf{u},-x) & (\mathbf{u}, x) \mapsto\left(\mathbf{w}, t_{3} x\right) & (\mathbf{u}, x) \mapsto\left(\mathbf{w}, t_{4} x\right) \\
(\mathbf{v}, x) \mapsto(\mathbf{v},-x) & (\mathbf{v}, x) \mapsto\left(\mathbf{x}, t_{3} x\right) & (\mathbf{v}, x) \mapsto\left(\mathbf{x}, t_{4} x\right) \\
(\mathbf{w}, x) \mapsto(\mathbf{x},-x) & (\mathbf{w}, x) \mapsto\left(\mathbf{u}, t_{3} x\right) & (\mathbf{w}, x) \mapsto\left(\mathbf{u}, t_{4} x\right) \\
(\mathbf{x}, x) \mapsto(\mathbf{w},-x), & (\mathbf{x}, x) \mapsto\left(\mathbf{v}, t_{3} x\right), & (\mathbf{x}, x) \mapsto\left(\mathbf{v}, t_{4} x\right)
\end{array}
$$

are isomorphisms from $\mathcal{C K}\left(2^{m}, 2,2^{m-2}+1\right)$ to $\mathcal{C K}\left(2^{m}, 2,2^{m-2}-1\right), \mathcal{C K}\left(2^{m}, 2\right.$, $\left.2^{m-2}+1\right)$ to $\mathcal{C K}\left(2^{m}, 2,2^{m-2}+2^{m-1}+1\right)$, and $\mathcal{C K}\left(2^{m}, 2,2^{m-2}-1\right)$ to $\mathcal{C K}\left(2^{m}, 2\right.$, $\left.2^{m-2}+2^{m-1}-1\right)$, respectively, where $x \in \mathbb{Z}_{2^{m}}$.

Note that the graphs $\mathcal{C K}\left(2^{m}, 1, k\right)$ and $\mathcal{C K}\left(2^{m}, 2, k\right)$ are denoted by $\mathcal{C K}\left(2^{m}, 1\right)$ and $\mathcal{C K}\left(2^{m}, 2\right)$ respectively in Theorem 2.1 , where $k=2^{m-2} \pm 1$ or $2^{m-2}+2^{m-1} \pm 1$. It will be shown that $\mathcal{C K}\left(2^{m}, 1\right)$ is not isomorphic to $\mathcal{C K}\left(2^{m}, 2\right)$ in Lemma 5.2.

\section{The proof of Theorem 2.1}

Let $\tilde{X}=K_{4,4} \times{ }_{\phi} \mathbb{Z}_{p^{m}}$ be a connected regular covering of the graph $K_{4,4}$ satisfying the hypotheses in Theorem 2.1, where $\phi=0$ on a spanning tree $T$ as illustrated by dark lines in Fig. 1 and we assign voltages $z_{1}, z_{2}, z_{3}, z_{4}, z_{5}, z_{6}, z_{7}, z_{8}$ and $z_{9}$ to the cotree arcs of $K_{4,4}$, where $z_{i} \in \mathbb{Z}_{p}^{m}$ for $1 \leq i \leq 9$. Thus, the fibre-preserving 
group contains a $\frac{1}{2}$-arc-transitive subgroup of $\operatorname{Aut}\left(K_{4,4} \times_{\phi} \mathbb{Z}_{p^{m}}\right)$. Hence, the projection of this subgroup is $\frac{1}{2}$-arc-transitive on the base graph $K_{4,4}$ and so a minimal $\frac{1}{2}$-arc-transitive subgroup, say $L$, of $K_{4,4}$ lifts. Since $K_{4,4} \times_{\phi} \mathbb{Z}_{p^{m}}$ is assumed to be connected, we have $\left\langle z_{1}, z_{2}, z_{3}, z_{4}, z_{5}, z_{6}, z_{7}, z_{8}, z_{9}\right\rangle=\mathbb{Z}_{p^{m}}$. Note that the vertex set of $K_{4,4}$ is $\{\mathbf{a}, \mathbf{b}, \mathbf{c}, \mathbf{d}, \mathbf{u}, \mathbf{v}, \mathbf{w}, \mathbf{x}\}$.

Possibly with aid of computer software package like MAGMA [3], or by a direct analysis of half-arc-transitive subgroups of $\operatorname{Aut}\left(K_{4,4}\right) \cong\left(S_{4} \times S_{4}\right) \rtimes \mathbb{Z}_{2}$, one can show that a minimal $\frac{1}{2}$-arc-transitive subgroup of $\operatorname{Aut}\left(K_{4,4}\right)$ is conjugate to one of $G_{1}=\left\langle\alpha_{1}, \beta_{1}\right\rangle, G_{2}=\left\langle\alpha_{1}, \beta_{2}\right\rangle$ and $G_{3}=\left\langle\alpha_{2}, \beta_{1}\right\rangle$, where $\alpha_{1}=(\mathbf{a} \mathbf{u} \mathbf{c} \mathbf{w} \mathbf{b} \mathbf{~ d ~} \mathbf{x})$, $\alpha_{2}=(\mathbf{a} \mathbf{u} \mathbf{c} \mathbf{w})(\mathbf{b} \mathbf{v} \mathbf{d} \mathbf{x}), \beta_{1}=(\mathbf{u} \mathbf{v})(\mathbf{w} \mathbf{x})$ and $\beta_{2}=(\mathbf{u} \mathbf{w})(\mathbf{v} \mathbf{x})(\mathbf{c} \mathbf{d})$. In each case, the stabilizer of $\mathbf{u}$ is isomorphic to $\mathbb{Z}_{2}$. Set $\gamma_{1}=(\mathbf{u} \mathbf{w} \mathbf{v} \mathbf{x})(\mathbf{c} \mathbf{d}), \gamma_{2}=(\mathbf{u} \mathbf{w} \mathbf{v} \mathbf{x})$ and $\gamma_{3}=$ $(\mathbf{u} \mathbf{w})(\mathbf{v} \mathbf{x})$. Clearly, all $\alpha_{i}, \beta_{j}$, and $\gamma_{k}(i, j=1,2 ; k=1,2,3)$ are automorphisms of $K_{4,4}$.

For $g \in \operatorname{Aut}\left(K_{4,4}\right)$, let $K_{4,4} \times_{\psi} \mathbb{Z}_{p^{m}}$ be the regular covering of $K_{4,4}$ derived by a voltage assignment $\psi$ defined by $\psi\left((\mathbf{y}, \mathbf{z})^{g}\right)=\phi((\mathbf{y}, \mathbf{z}))$ for each $\operatorname{arc}(\mathbf{y}, \mathbf{z}) \in$ $A\left(K_{4,4}\right)$. Clearly, $\psi$ is $T^{g}$-reduced ( $T^{g}$ is the image of the spanning tree $T$ under $g$ ) and $\widetilde{g}:(\mathbf{z}, x) \mapsto\left(\mathbf{z}^{g}, x\right)$ is an isomorphism from $\widetilde{X}=K_{4,4} \times_{\phi} \mathbb{Z}_{p^{m}}$ to $K_{4,4} \times_{\psi} \mathbb{Z}_{p^{m}}$, where $\mathbf{z} \in V\left(K_{4,4}\right)$ and $x \in \mathbb{Z}_{p^{m}}$. By Proposition 3.1, L lifts in $K_{4,4} \times_{\phi} \mathbb{Z}_{p^{m}}$ if and only if $L^{g}$ lifts in $K_{4,4} \times_{\psi} \mathbb{Z}_{p^{m}}$ (see also [24, Proposition 2.1]). Thus, to classify $\frac{1}{2}$-arc-transitive cyclic coverings of $K_{4,4}$, one may assume that one of the minimal $\frac{1}{2}$-arc-transitive subgroups $G_{1}, G_{2}$ and $G_{3}$ lifts.

Denote by $i_{1} i_{2} \cdots i_{s}$ the cycle having the consecutively adjacent vertices $i_{1}, i_{2}, \ldots, i_{s}$. There are nine fundamental cycles ubva, wbva, xbva, ucwa, vcwa, xcwa, udxa, vdxa and wdxa, which are generated by the nine cotree $\operatorname{arcs}(\mathbf{u}, \mathbf{b})$, $(\mathbf{w}, \mathbf{b}),(\mathbf{x}, \mathbf{b}),(\mathbf{u}, \mathbf{c}),(\mathbf{v}, \mathbf{c}),(\mathbf{x}, \mathbf{c}),(\mathbf{u}, \mathbf{d}),(\mathbf{v}, \mathbf{d})$ and $(\mathbf{w}, \mathbf{d})$, respectively. Each cycle maps to a cycle of the same length under the actions of $\alpha_{1}, \alpha_{2}, \beta_{1}, \beta_{2}, \gamma_{1}, \gamma_{2}$, and $\gamma_{3}$. We list these cycles and their voltages in Tables 1 and 2, in which $C$ denotes a fundamental cycle of $K_{4,4}$ and $\phi(C)$ denotes the voltage on $C$.

Consider the mapping $\bar{\alpha}_{1}$ from the set of voltages of the nine fundamental cycles of $K_{4,4}$ to the cyclic group $\mathbb{Z}_{p^{m}}$ defined by $\phi(C)^{\bar{\alpha}_{1}}=\phi\left(C^{\alpha_{1}}\right)$, where $C$ runs over the nine fundamental cycles. Similarly, we can define $\bar{\alpha}_{2}, \bar{\beta}_{1}, \bar{\beta}_{2}, \bar{\gamma}_{1}, \bar{\gamma}_{2}$ and $\bar{\gamma}_{3}$. By Proposition 3.1, if $\alpha_{1}$ lifts then $\bar{\alpha}_{1}$ can be extended to an automorphism of $\mathbb{Z}_{p^{m}}$

Table 1 Voltages on fundamental cycles and their images under $\alpha_{1}, \beta_{1}$ and $\gamma_{1}$

\begin{tabular}{llllllll}
\hline$C$ & $\phi(C)$ & $C^{\alpha_{1}}$ & $\phi\left(C^{\alpha_{1}}\right)$ & $C^{\beta_{1}}$ & $\phi\left(C^{\beta_{1}}\right)$ & $C^{\gamma_{1}}$ & $\phi\left(C^{\gamma_{1}}\right)$ \\
\hline ubva & $z_{1}$ & cvdu & $-z_{5}+z_{8}-z_{7}+z_{4}$ & vbua & $-z_{1}$ & wbxa & $z_{2}-z_{3}$ \\
wbva & $z_{2}$ & bvdu & $z_{8}-z_{7}+z_{1}$ & xbua & $z_{3}-z_{1}$ & vbxa & $-z_{3}$ \\
xbva & $z_{3}$ & avdu & $z_{8}-z_{7}$ & wbua & $z_{2}-z_{1}$ & ubxa & $z_{1}-z_{3}$ \\
ucwa & $z_{4}$ & cwbu & $z_{2}-z_{1}+z_{4}$ & vcxa & $z_{5}-z_{6}$ & wdva & $z_{9}-z_{8}$ \\
vcwa & $z_{5}$ & dwbu & $-z_{9}+z_{2}-z_{1}+z_{7}$ & ucxa & $z_{4}-z_{6}$ & xdva & $-z_{8}$ \\
xcwa & $z_{6}$ & awbu & $z_{2}-z_{1}$ & wcxa & $-z_{6}$ & udva & $z_{7}-z_{8}$ \\
udxa & $z_{7}$ & cxau & $-z_{6}+z_{4}$ & vdwa & $z_{8}-z_{9}$ & wcua & $-z_{4}$ \\
vdxa & $z_{8}$ & dxau & $z_{7}$ & udwa & $z_{7}-z_{9}$ & xcua & $z_{6}-z_{4}$ \\
wdxa & $z_{9}$ & bxau & $-z_{3}+z_{1}$ & xdwa & $-z_{9}$ & vcua & $z_{5}-z_{4}$ \\
\hline
\end{tabular}


Table 2 Voltages on fundamental cycles and their images under $\alpha_{2}, \beta_{2}, \gamma_{2}$ and $\gamma_{3}$

\begin{tabular}{llllllll}
\hline$C^{\alpha_{2}}$ & $\phi\left(C^{\alpha_{2}}\right)$ & $C^{\beta_{2}}$ & $\phi\left(C^{\beta_{2}}\right)$ & $C^{\gamma_{2}}$ & $\phi\left(C^{\gamma_{2}}\right)$ & $C^{\gamma_{3}}$ & $\phi\left(C^{\gamma_{3}}\right)$ \\
\hline cvdu & $-z_{5}+z_{8}-z_{7}+z_{4}$ & wbxa & $z_{2}-z_{3}$ & wbxa & $z_{2}-z_{3}$ & wbxa & $z_{2}-z_{3}$ \\
avdu & $z_{8}-z_{7}$ & ubxa & $z_{1}-z_{3}$ & vbxa & $-z_{3}$ & ubxa & $z_{1}-z_{3}$ \\
bvdu & $z_{8}-z_{7}+z_{1}$ & vbxa & $-z_{3}$ & ubxa & $z_{1}-z_{3}$ & vbxa & $-z_{3}$ \\
cwau & $z_{4}$ & wdua & $z_{9}-z_{7}$ & wcva & $-z_{5}$ & wcua & $-z_{4}$ \\
dwau & $-z_{9}+z_{7}$ & xdua & $-z_{7}$ & xcva & $z_{6}-z_{5}$ & xcua & $z_{6}-z_{4}$ \\
bwau & $-z_{2}+z_{1}$ & vdua & $z_{8}-z_{7}$ & ucva & $z_{4}-z_{5}$ & vcua & $z_{5}-z_{4}$ \\
cxbu & $-z_{6}+z_{3}-z_{1}+z_{4}$ & wcva & $-z_{5}$ & wdua & $z_{9}-z_{7}$ & wdva & $z_{9}-z_{8}$ \\
dxbu & $z_{3}-z_{1}+z_{7}$ & xcva & $z_{6}-z_{5}$ & xdua & $-z_{7}$ & xdva & $-z_{8}$ \\
axbu & $z_{3}-z_{1}$ & ucva & $z_{4}-z_{5}$ & vdua & $z_{8}-z_{7}$ & udva & $z_{7}-z_{8}$ \\
\hline
\end{tabular}

and in this case, denote by $\alpha_{1}^{*}$ the extended automorphism. Clearly, there is a homomorphism from the generated group $\left\langle\alpha_{1}\right\rangle$ to $\mathbb{Z}_{p^{m}}^{*}$ defined by $\alpha_{1}^{i} \mapsto\left(\alpha_{1}^{*}\right)^{i}$ for any $i$. Since $\alpha_{1}$ has order 8 , we have $\left(\alpha_{1}^{*}\right)^{8}=1$. Similarly, one has the notation $\alpha_{2}^{*}, \beta_{1}^{*}, \beta_{2}^{*}, \gamma_{1}^{*}, \gamma_{2}^{*}$ and $\gamma_{3}^{*}$ if they lift. Since $\alpha_{2}, \beta_{1}, \beta_{2}$ have orders $4,2,2$, we have $\left(\alpha_{2}^{*}\right)^{4}=\left(\beta_{1}^{*}\right)^{2}=\left(\beta_{2}^{*}\right)^{2}=1$. To finish the proof of Theorem 2.1, we consider three cases separately, depending on whether $G_{1}, G_{2}$, or $G_{3}$ lifts.

Lemma 5.1 If $G_{1}$ lifts and $\widetilde{X}$ is $\frac{1}{2}$-arc-transitive then $p=2, m \geq 4$ and $\widetilde{X} \cong$ $\mathcal{C K}\left(2^{m}, 1\right)$ or $\mathcal{C K}\left(2^{m}, 2\right)$.

Proof Since $G_{1}=\left\langle\alpha_{1}, \beta_{1}\right\rangle$ lifts, $\alpha_{1}^{*}$ and $\beta_{1}^{*}$ exist. Let $1^{\alpha_{1}{ }^{*}}=k$. Then $k \in \mathbb{Z}_{p^{m}}^{*}$. By Table $1, k z_{8}=z_{8}^{\alpha_{1}^{*}}=z_{7}$, that is, $z_{7}=k z_{8}$. Thus, $k z_{3}=z_{3}^{\alpha_{1}^{*}}=z_{8}-z_{7}$ implies that $z_{3}=\left(k^{-1}-1\right) z_{8}$, where $k^{-1}$ is the inverse of $k$ in $\mathbb{Z}_{p^{m}}^{*}$. Similarly, by considering the images of $z_{2}, z_{6}, z_{7}, z_{9}$ and $z_{5}$ under $\alpha_{1}^{*}$ respectively, one may obtain that $z_{1}, z_{6}, z_{4}$, $z_{9}$ and $z_{5}$ are combinations of $z_{2}$ and $z_{8}$. Thus, we have Eqs. 1-7 which means that $\mathbb{Z}_{p^{m}}$ is generated by $z_{2}$ and $z_{8}$. Since $k z_{4}=z_{4}^{\alpha_{1}^{*}}=z_{2}-z_{1}+z_{4}$, by Eqs. 1 and 3 , one may obtain Eq. 8. Since $\beta_{1}^{*}$ exists, one has $1^{\beta_{1}{ }^{*}}=s$ for some $s \in \mathbb{Z}_{p^{m}}^{*}$. By Table 1 , $s z_{1}=-z_{1}, s z_{2}=z_{3}-z_{1}, s z_{3}=z_{2}-z_{1}, s z_{4}=z_{5}-z_{6}, s z_{5}=z_{4}-z_{6}, s z_{6}=-z_{6}$, $s z_{7}=z_{8}-z_{9}, s z_{8}=z_{7}-z_{9}$ and $s z_{9}=-z_{9}$. Since $\left(\beta_{1}^{*}\right)^{2}=1$, one has $s^{2}=1$ in the $\operatorname{ring} \mathbb{Z}_{p^{m}}$.

$$
\begin{aligned}
& z_{1}=k z_{2}+(k-1) z_{8} \\
& z_{3}=\left(k^{-1}-1\right) z_{8} \\
& z_{4}=\left(k^{-1}-1\right) z_{2}+\left(k^{2}+k^{-1}-1\right) z_{8} \\
& z_{5}=-z_{2}+k^{-3} z_{8} \\
& z_{6}=\left(k^{-1}-1\right) z_{2}+\left(k^{-1}-1\right) z_{8} \\
& z_{7}=k z_{8} \\
& z_{9}=z_{2}+\left(1-k^{-2}\right) z_{8} \\
& (k-1) z_{2}=-(k-1)\left(k^{3}+1\right) z_{8} .
\end{aligned}
$$


Substituting Eqs. 6 and 7 to the equation $s z_{8}=z_{7}-z_{9}$, one has $z_{2}=\left(k+k^{-2}-\right.$ $s-1) z_{8}$, implying that $\mathbb{Z}_{p^{m}}$ can be generated by $z_{8}$. By Proposition 3.2, one may assume that $z_{8}=1$ and so $z_{2}=k+k^{-2}-s-1$. Suppose $\beta_{2}$ lifts. Then the arctransitive subgroup $\left\langle\alpha_{1}, \beta_{1}, \beta_{2}\right\rangle$ of $K_{4,4}$ lifts, contradicting half-arc-transitivity of $\widetilde{X}$. By Proposition 3.1, $\bar{\beta}_{2}$ cannot be extended to an automorphism of $\mathbb{Z}_{p^{m}}$. Similarly, $\bar{\gamma}_{2}$ also cannot be extended to an automorphism of $\mathbb{Z}_{p^{m}}$.

Assume that $p$ is odd. Then $s^{2}=1$ implies that $s=1$ or -1 because $\mathbb{Z}_{p^{m}}^{*}$ is cyclic. Suppose $s=1$. Then $z_{2}=k+k^{-2}-2$ and $z_{8}=1$. Since $s z_{9}=-z_{9}$, we have $2 z_{9}=0$, forcing that $z_{9}=0$ because $p$ is odd. By Eq. 7, $k=1$ and so $z_{2}=0$ and $z_{8}=1$. By Eqs. $1-7, z_{1}=z_{3}=z_{6}=z_{9}=0$ and $z_{4}=z_{5}=z_{7}=1$. By Table 2, $\bar{\beta}_{2}$ can be extended to the automorphism of $\mathbb{Z}_{p^{m}}$ induced by $1 \mapsto-1$, a contradiction. Thus, $s=-1$. Then $z_{2}=k+k^{-2}-s-1=k+k^{-2}$ and $z_{8}=1$. Furthermore, $s z_{2}=z_{3}-z_{1}$ means that $z_{1}=z_{2}+z_{3}$. Substituting Eqs. 1 and 2 to $z_{1}=z_{2}+z_{3}$, one has $k^{4}=1$. It follows that $k^{2}=1$ or $k^{2}=-1$ because $\mathbb{Z}_{p^{m}}^{*}$ is cyclic. If $k^{2}=1$ then $z_{2}=k+1$ and $z_{8}=1$. By Eqs. 1-7, one has $z_{1}=2 k, z_{3}=k-1, z_{4}=k, z_{5}=-1, z_{6}=k-1$, $z_{7}=k$ and $z_{9}=k+1$. By Table $2, \bar{\beta}_{2}$ can be extended to the automorphism of $\mathbb{Z}_{p^{m}}$ induced by $1 \mapsto k$, contrary to our hypothesis. Similarly, if $k^{2}=-1$ then one may have that $z_{1}=-2, z_{2}=k-1, z_{3}=-k-1, z_{4}=-k, z_{5}=1, z_{6}=-k+1, z_{7}=k$, $z_{8}=1$ and $z_{9}=k+1$. By Table $2, \bar{\gamma}_{2}$ can be extended to the automorphism of $\mathbb{Z}_{p^{m}}$ induced by $1 \mapsto-k$, a contradiction.

Now assume that $p=2$. For $m=1,|V(\widetilde{X})|=16$ and since the smallest $\frac{1}{2}$-arctransitive graph has order 27 [46], we have $m \geq 2$. Then $s^{2}=1$ implies that $s= \pm 1$ or $2^{m-1} \pm 1$ because $\mathbb{Z}_{2^{m}}^{*} \cong \mathbb{Z}_{2} \times \mathbb{Z}_{2^{m-2}}$. Thus, $s=\delta+1$ or $\delta-1$, where $\delta=0$ or $2^{m-1}$. Suppose $s=\delta+1$. Then $z_{8}=1$ and $z_{2}=k+k^{-2}+\delta-2$. Since $k \in \mathbb{Z}_{2^{m}}^{*}, k$ is odd and so $z_{2}$ is even. By Eq. 7, $z_{9}$ is even. Since $s z_{9}=-z_{9}, s=\delta+1$ implies that $2 z_{9}=0$. It follows that $z_{9}=0$ or $2^{m-1}$. Write $z_{9}=\delta_{1}$ where $\delta_{1}=0$ or $2^{m-1}$. Again by Eq. $7, z_{2}=k^{-2}+\delta_{1}-1$. Since $z_{2}=k+k^{-2}+\delta-2$, one has $k=\delta+\delta_{1}+1$. In this case, $z_{2}=\delta_{1}$ and $z_{8}=1$. Since $k$ is odd, $k \delta=\delta$ and $k \delta_{1}=\delta_{1}$, and by Eqs. $1-7$, $z_{1}=\delta, z_{3}=\delta+\delta_{1}, z_{4}=\delta+\delta_{1}+1, z_{5}=\delta+1, z_{6}=\delta+\delta_{1}, z_{7}=\delta+\delta_{1}+1$ and $z_{9}=\delta_{1}$. By Table $2, \bar{\beta}_{2}$ can be extended to the automorphism of $\mathbb{Z}_{2^{m}}$ induced by $1 \mapsto \delta_{1}-1$, a contradiction. Thus, $s=\delta-1$.

We have $z_{8}=1$ and $z_{2}=k+k^{-2}-s-1=k+k^{-2}+\delta$ when $s=\delta-1$. Recall that $k$ is odd and so $k \delta=\delta$. Substituting Eqs. 1 and 2 to $s z_{2}=z_{3}-z_{1}$, one has $k^{4}=1$. Since $\mathbb{Z}_{2^{m}}^{*} \cong \mathbb{Z}_{2} \times \mathbb{Z}_{2^{m-2}}(m \geq 2)$, if $m=2$ or 3 then $k^{2}=1$ and if $m \geq 3$ then $k= \pm 1,2^{m-1} \pm 1,2^{m-2} \pm 1$ or $2^{m-2}+2^{m-1} \pm 1$, implying that $k^{2}=1$ or $k^{2}=2^{m-1}+1$. Suppose $k^{2}=1$. Then $z_{2}=k+\delta+1$ and $z_{8}=1$. By Eqs. $1-7$, one has $z_{1}=2 k+\delta, z_{3}=k-1, z_{4}=k, z_{5}=\delta-1, z_{6}=k-1, z_{7}=k$ and $z_{9}=k+\delta+1$. By Table $2, \bar{\beta}_{2}$ can be extended to the automorphism of $\mathbb{Z}_{2^{m}}$ induced by $1 \mapsto k+\delta$, a contradiction. Thus, one may assume that $m \geq 4$ and $k^{2}=2^{m-1}+1$, forcing that $k$ is an element of order 4 in $\mathbb{Z}_{2^{m}}^{*}$. In this case, $z_{8}=1$ and $z_{2}=k+k^{-2}-s-1=$ $k+2^{m-1}+\delta+1$. By Eqs. $1-7$, one has $z_{1}=2 k+\delta, z_{3}=k+2^{m-1}-1, z_{4}=k+2^{m-1}$, $z_{5}=2^{m-1}+\delta-1, z_{6}=k-1, z_{7}=k$ and $z_{9}=k+\delta+1$. By the constructions of graphs in Sect. $1, \widetilde{X} \cong \mathcal{C K}\left(2^{m}, \underset{\widetilde{X}}{1}, k\right)$ when $\delta=0$ and $\widetilde{X} \cong \mathcal{C K}\left(2^{m}, 2, k\right)$ when $\delta=2^{m-1}$. Thus, $\widetilde{\widetilde{X}} \cong \mathcal{C K}\left(2^{m}, 1\right)$ or $\widetilde{X} \cong \mathcal{C K}\left(2^{m}, 2\right)$ by Lemma 4.1 .

Lemma $5.2 \mathcal{C K}\left(2^{m}, 1\right)$ and $\mathcal{C K}\left(2^{m}, 2\right)$ are non-isomorphic $\frac{1}{2}$-arc-transitive graphs for each $m \geq 4$. Furthermore, these graphs are of even radius and tightly attached. 
Proof By the proof of Lemma 5.1, it suffices to show that $\mathcal{C K}\left(2^{m}, 1,2^{m-2}+1\right)$ and $\mathcal{C K}\left(2^{m}, 2,2^{m-2}+1\right)$ are non-isomorphic $\frac{1}{2}$-arc-transitive graphs, which are of even radius and tightly attached. Let $\tilde{X}=\mathcal{C} \mathcal{K}\left(2^{m}, 1,2^{m-2}+1\right)$ or $\mathcal{C K}\left(2^{m}, 2,2^{m-2}+1\right)$,

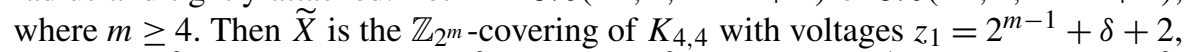
$z_{2}=-2^{m-2}+\delta+2, z_{3}=-2^{m-2}, z_{4}=-2^{m-2}+1, z_{5}=2^{m-1}+\delta-1, z_{6}=2^{m-2}$, $z_{7}=2^{m-2}+1, z_{8}=1$ and $z_{9}=2^{m-2}+\delta+2$, where $\delta=0$ corresponds to the graph $\mathcal{C K}\left(2^{m}, 1,2^{m-2}+1\right)$ and $\delta=2^{m-1}$ corresponds to the graph $\mathcal{C K}\left(2^{m}, 2,2^{m-2}+1\right)$. By Table $1, \bar{\alpha}_{1}$ and $\bar{\beta}_{1}$ can be extended to the automorphisms of $\mathbb{Z}_{2^{m}}$ induced by $1 \mapsto$ $2^{m-2}+1$ and $1 \mapsto \delta-1$ respectively. Suppose $\beta_{2}$ lifts. Then $\beta_{2}^{*}$ exists. By Table 2 , $z_{8}^{\beta_{2}^{*}}=1^{\beta_{2}^{*}}=z_{6}-z_{5}=-2^{m-2}+\delta+1$ and $z_{7}^{\beta_{2}^{*}}=\left(2^{m-2}+1\right)^{\beta_{2}^{*}}=-z_{5}=2^{m-1}+\delta+1$. This is impossible because $\left(2^{m-2}+1\right)^{\beta_{2}^{*}}=\left(-2^{m-2}+\delta+1\right)\left(2^{m-2}+1\right)=\delta+1$. Thus, $\beta_{2}$ does not lift. Since $G_{1}=\left\langle\alpha_{1}, \beta_{1}\right\rangle$ is $\frac{1}{2}$-arc-transitive on $K_{4,4}$, the lifting group of $G_{1}$, say $B$, is $\frac{1}{2}$-arc-transitive on $\widetilde{X}$. Clearly, $B \leq \operatorname{Aut}(\tilde{X})$ and since $\left|G_{1}\right|=2^{4}$, one has $|B|=2^{m+4}$. Moreover, the stabilizer $B_{(\mathbf{z}, x)}$ of a vertex $(\mathbf{z}, x) \in V(\widetilde{X})$ in $B$ is isomorphic to $\mathbb{Z}_{2}$.

For convenience, we use $\mathbf{z}_{x}$ to denote the vertex $(\mathbf{z}, x)$ of $\tilde{X}$ for $\mathbf{z} \in V\left(K_{4,4}\right)$ and $x \in \mathbb{Z}_{2^{m}}$. It is easy to show that the numbers of vertices at distance 2 and 3 from $\mathbf{a}_{0}$ in $\widetilde{X}$ are 12 and 36 respectively. Thus, $\widetilde{X}$ has girth at least 8 because $\widetilde{X}$ is bipartite. Let $T_{1}, T_{2}, T_{3}$ and $T_{4}$ be the sets of vertices passing through the $\operatorname{arcs}\left(\mathbf{a}_{0}, \mathbf{u}_{0}\right),\left(\mathbf{a}_{0}, \mathbf{v}_{0}\right)$, $\left(\mathbf{a}_{0}, \mathbf{w}_{0}\right)$ and $\left(\mathbf{a}_{0}, \mathbf{x}_{0}\right)$ respectively, and having distance 4 from the vertex $\mathbf{a}_{0}$ in $\widetilde{X}$. One may compute the following:

$$
\begin{aligned}
& T_{1}=\left\{\mathbf{a}_{2^{m-1}+\delta+2}, \mathbf{a}_{-2^{m-2}}, \mathbf{a}_{-2^{m-2}+\delta+2}, \mathbf{a}_{2^{m-2}+\delta+2}, \mathbf{a}_{-2^{m-2}+1}, \mathbf{a}_{2^{m-1}+1}, \mathbf{a}_{2^{m-2}}, \mathbf{a}_{\delta-1},\right. \\
& \mathbf{a}_{2^{m-2}+1}, \mathbf{b}_{2^{m-2}+\delta+2}, \mathbf{b}_{2^{m-1}+\delta+3}, \mathbf{b}_{2^{m-2}+1}, \mathbf{b}_{2^{m-2}}, \mathbf{b}_{-2^{m-2}+1}, \mathbf{b}_{1}, \mathbf{c}_{1}, \mathbf{c}_{-2^{m-2}}, \\
& \mathbf{c}_{\delta+2}, \mathbf{c}_{-2^{m-2}+\delta-1}, \mathbf{c}_{\delta-1}, \mathbf{c}_{2^{m-1}+1}, \mathbf{d}_{2^{m-1}+\delta+3}, \mathbf{d}_{\delta+2}, \mathbf{d}_{-2^{m-2}+\delta+2}, \mathbf{d}_{2^{m-2}+\delta+3}, \\
& \left.\mathbf{d}_{\delta+3}, \mathbf{d}_{2^{m-1}+1}\right\} \text {, } \\
& T_{2}=\left\{\mathbf{a}_{2^{m-1}+\delta-2}, \mathbf{a}_{2^{m-2}+\delta-2}, \mathbf{a}_{2^{m-2}}, \mathbf{a}_{-2^{m-2}+\delta-2}, \mathbf{a}_{2^{m-1}+\delta-1}, \mathbf{a}_{2^{m-2}+\delta-1}, \mathbf{a}_{-2^{m-2}},\right. \\
& \mathbf{a}_{-2^{m-2}+\delta-1}, \mathbf{a}_{1}, \mathbf{b}_{2^{m-2}}, \mathbf{b}_{2^{m-2}+1}, \mathbf{b}_{\delta-1}, \mathbf{b}_{2^{m-2}+\delta+2}, \mathbf{b}_{2^{m-1}+1}, \mathbf{b}_{-2^{m-2}+1}, \\
& \mathbf{c}_{2^{m-2}+\delta-1}, \mathbf{c}_{2^{m-2}+\delta-2}, \mathbf{c}_{2^{m-1}}, \mathbf{c}_{2^{m-1}+1}, \mathbf{c}_{-2^{m-2}+\delta-1}, \mathbf{c}_{2^{m-2}+1}, \mathbf{d}_{-2^{m-2}+\delta-1}, \\
& \left.\mathbf{d}_{2^{m-1}}, \mathbf{d}_{2^{m-2}}, \mathbf{d}_{\delta-1}, \mathbf{d}_{-2^{m-2}+1}, \mathbf{d}_{2^{m-2}+\delta-1}\right\} \text {, } \\
& T_{3}=\left\{\mathbf{a}_{2^{m-2}}, \mathbf{a}_{-2^{m-2}+\delta+2}, \mathbf{a}_{\delta+2}, \mathbf{a}_{2^{m-2}-1}, \mathbf{a}_{2^{m-1}+\delta+1}, \mathbf{a}_{-2^{m-2}}, \mathbf{a}_{\delta+1}, \mathbf{a}_{2^{m-2}+\delta+1},\right. \\
& \mathbf{a}_{2^{m-2}+\delta+2}, \mathbf{b}_{-2^{m-2}+\delta+1}, \mathbf{b}_{2^{m-1}+\delta+1}, \mathbf{b}_{2^{m-1}}, \mathbf{b}_{2^{m-1}+3}, \mathbf{b}_{2^{m-2}+\delta+1}, \mathbf{b}_{\delta+2}, \mathbf{c}_{1}, \\
& \mathbf{c}_{2^{m-2}+1}, \mathbf{c}_{-2^{m-2}+\delta+2}, \mathbf{c}_{2^{m-2}+\delta+2}, \mathbf{c}_{-2^{m-2}}, \mathbf{c}_{2^{m-1}+\delta+2}, \mathbf{d}_{2^{m-1}+1}, \mathbf{d}_{-2^{m-2}+\delta+3}, \\
& \left.\mathbf{d}_{\delta+2}, \mathbf{d}_{2^{m-1}}, \mathbf{d}_{2^{m-1}+\delta+2}, \mathbf{d}_{-2^{m-2}}\right\} \text {, } \\
& T_{4}=\left\{\mathbf{a}_{2^{m-2}+\delta-2}, \mathbf{a}_{-2^{m-2}}, \mathbf{a}_{\delta-2}, \mathbf{a}_{2^{m-1}-1}, \mathbf{a}_{-2^{m-2}+\delta+1}, \mathbf{a}_{2^{m-2}}, \mathbf{a}_{-2^{m-2}-1}, \mathbf{a}_{-1},\right. \\
& \mathbf{a}_{-2^{m-2}+\delta-2}, \mathbf{b}_{\delta+1}, \mathbf{b}_{-2^{m-2}+\delta+1}, \mathbf{b}_{\delta+2}, \mathbf{b}_{2^{m-2}+\delta+1}, \mathbf{b}_{-1}, \mathbf{b}_{2^{m-1}}, \mathbf{c}_{\delta-1}, \mathbf{c}_{2^{m-2}+\delta-1}, \\
& \mathbf{c}_{\delta-2}, \mathbf{c}_{2^{m-1}+\delta-2}, \mathbf{c}_{2^{m-1}}, \mathbf{c}_{-2^{m-2}+\delta-2}, \mathbf{d}_{2^{m-1}+\delta-1}, \mathbf{d}_{-2^{m-2}+1}, \mathbf{d}_{2^{m-2}}, \mathbf{d}_{-2^{m-2}}, \\
& \left.\mathbf{d}_{-2^{m-2}+\delta+2}, \mathbf{d}_{2^{m-1}+\delta+2}\right\} \text {. }
\end{aligned}
$$

Furthermore, one may obtain that if $\widetilde{X}=\mathcal{C K}\left(2^{m}, 1,2^{m-2}+1\right)(\delta=0)$ then

$$
\begin{array}{lll}
\text { for } m \geq 5, & \left|T_{1} \cap T_{2}\right|=\left|T_{3} \cap T_{4}\right|=8, \quad\left|T_{1} \cap T_{3}\right|=\left|T_{2} \cap T_{4}\right|=13, \\
& \left|T_{1} \cap T_{4}\right|=\left|T_{2} \cap T_{3}\right|=9 ; \\
\text { for } m=4, & \left|T_{1} \cap T_{2}\right|=\left|T_{3} \cap T_{4}\right|=\left|T_{1} \cap T_{3}\right|=\left|T_{2} \cap T_{4}\right|=13, \\
& \left|T_{1} \cap T_{4}\right|=\left|T_{2} \cap T_{3}\right|=12 .
\end{array}
$$


If $\tilde{X}=\mathcal{C K}\left(2^{m}, 2,2^{m-2}+1\right)\left(\delta=2^{m-1}\right)$ then

$$
\begin{aligned}
& \text { for } m \geq 5, \quad\left|T_{1} \cap T_{2}\right|=\left|T_{3} \cap T_{4}\right|=\left|T_{1} \cap T_{4}\right|=\left|T_{2} \cap T_{3}\right|=8, \\
& \left|T_{1} \cap T_{3}\right|=\left|T_{2} \cap T_{4}\right|=13 ; \\
& \text { for } m=4, \quad\left|T_{1} \cap T_{2}\right|=\left|T_{3} \cap T_{4}\right|=\left|T_{1} \cap T_{3}\right|=\left|T_{2} \cap T_{4}\right|=13 \text {, } \\
& \left|T_{1} \cap T_{4}\right|=\left|T_{2} \cap T_{3}\right|=11 .
\end{aligned}
$$

Thus, $\widetilde{X}$ has girth 8 and $\mathcal{C K}\left(2^{m}, 1,2^{m-2}+1\right) \neq \mathcal{C K}\left(2^{m}, 2,2^{m-2}+1\right)$ because if $m \geq 5,\left|T_{1} \cap T_{4}\right|=9$ in $\mathcal{C K}\left(2^{m}, 1,2^{m-2}+1\right)$ and for any $1 \leq i, j \leq 4,\left|T_{i} \cap T_{j}\right| \neq 9$ in $\bar{C} \mathcal{K}\left(2^{m}, 2,2^{m-2}+1\right)$; if $m=4,\left|T_{1} \cap T_{4}\right|=12$ in $\mathcal{C K}\left(2^{m}, 1,2^{m-2}+1\right)$ and for any $1 \leq i, j \leq 4,\left|T_{i} \cap T_{j}\right| \neq 12$ in $\mathcal{C K}\left(2^{m}, 2,2^{m-2}+1\right)$ (note that $\left|T_{1} \cap T_{4}\right|=9$ implies that there are exactly 9 cycles of length 8 passing through the two $\operatorname{arcs}\left(\mathbf{a}_{0}, \mathbf{u}_{0}\right)$ and $\left(\mathbf{a}_{0}, \mathbf{x}_{0}\right)$ in $\left.\mathcal{C K}\left(2^{m}, 1,2^{m-2}+1\right)\right)$.

Let $H=\operatorname{Aut}(\tilde{X})$. First we show that $H$ is a 2 -group. Since $\underset{\widetilde{X}}{\widetilde{X}}$ has valency $4, H_{\mathbf{a}_{0}}$ is a $\{2,3\}$-group. Suppose that $H$ is not a 2-group. Since $|V(\widetilde{X})|=2^{m+3},\left|H_{\mathbf{a}_{0}}\right|$ is divisible by 3 and the transitivity of $H$ implies that there is an element of order 3 , say $\alpha$, in $H_{\mathbf{a}_{0}}$ such that $\alpha$ has a 3-orbit on $\left\{\mathbf{u}_{0}, \mathbf{v}_{0}, \mathbf{w}_{0}, \mathbf{x}_{0}\right\}$, the neighborhood of $\mathbf{a}_{0}$ in $\widetilde{X}$. If $\alpha$ fixes $\mathbf{u}_{0}$ then $\left|T_{1} \cap T_{2}\right|=\left|T_{1} \cap T_{3}\right|=\left|T_{1} \cap T_{4}\right|$, which is not true for both graphs $\mathcal{C K}\left(2^{m}, 1,2^{m-2}+1\right)$ and $\mathcal{C K}\left(2^{m}, 2,2^{m-2}+1\right)$. One may obtain a similar contradiction if $\alpha$ fixes any given vertex in $\left\{\mathbf{u}_{0}, \mathbf{v}_{0}, \mathbf{w}_{0}, \mathbf{x}_{0}\right\}$. Thus, $H$ is a 2-group. Recall that $B$ is $\frac{1}{2}$-arc-transitive on $\widetilde{X}$ and $|B|=2^{m+4}$.

To prove half-arc-transitivity of $\widetilde{X}$, it suffices to show that $H=B$. Suppose to the contrary that $H \neq B$. Since $H$ is a 2-group, there is a subgroup $A$ of $H$ such that $B \leq A$ and $|A: B|=2$. Thus, $|A|=2^{m+5}$ and $B \triangleleft A$. Let $p_{1}$ be the projection corresponding to the regular covering $\widetilde{X}$ of $K_{4,4}$. Since $B$ is the lift of $G_{1}, B$ is fibrepreserving along $p_{1}$.

We claim that $A$ is fibre-preserving along $p_{1}$. Note that the covering transformation group $K=\mathbb{Z}_{2^{m}}(m \geq 4)$ is identified as an automorphism group of $\widetilde{X}$ by right multiplication on the second coordinate of each vertex in $\widetilde{X}$ (see the second paragraph in Sect. 3). Then $K \triangleleft B$ because $B$ is the lift of the group $G_{1}=\left\langle\alpha_{1}, \beta_{1}\right\rangle$. Since $|A: B|=2, K$ has at most two conjugacy classes in $A$, that is, $K$ and $K^{a}$ for some $a \in A$ (if the conjugacy class is only one then $K=K^{a}$ ). It follows that $K \cap K^{a} \triangleleft A$. Clearly, $K^{a} \triangleleft B$. Suppose $K K^{a}=B$. Then $B / K=K K^{a} / K \cong K^{a} / K \cap K^{a}$ is cyclic. On the other hand, $B / K \cong\left\langle\alpha_{1}, \beta_{1}\right\rangle$ is not cyclic, a contradiction. Thus, $K K^{a} \neq B$ and so $\left|K K^{a}\right|=2^{m+i}$ for some $0 \leq i \leq 3$ because $|B|=2^{m+4}$. It follows that $\left|K \cap K^{a}\right|=2^{m-i}$ and so $K \cap K^{a}=\left\langle 2^{i}\right\rangle$ because $K=\mathbb{Z}_{2^{m}}$ is cyclic. Since $K \cap K^{a}$ is cyclic, each of its subgroups is characteristic in $K \cap K^{a}$. Thus, each subgroup of $K \cap K^{a}$ is normal in $A$ because $K \cap K^{a} \triangleleft A$. Since $K \cap K^{a}=\left\langle 2^{i}\right\rangle$ for $0 \leq i \leq 3$, we always have $\left\langle 2^{3}\right\rangle \triangleleft A$. Let $Y$ be the quotient graph of $\widetilde{X}$ corresponding to the orbits of $\left\langle 2^{3}\right\rangle$. Write $\mathcal{A}_{i}=\left\{\mathbf{a}_{x+i} \mid x \in\left\langle 2^{3}\right\rangle\right\}$ for $i \in \mathbb{Z}_{8}$, and denote by $\mathcal{B}_{i}, \mathcal{C}_{i}, \mathcal{D}_{i}, \mathcal{U}_{i}, \mathcal{V}_{i}, \mathcal{W}_{i}$ and $\mathcal{X}_{i}$ the similar sets as $\mathcal{A}_{i}$. Then these sets are the orbits of $\left\langle 2^{3}\right\rangle$ and so the vertices of $Y$. Recall that $z_{1}=2^{m-1}+\delta+2, z_{2}=-2^{m-2}+\delta+2 z_{3}=-2^{m-2}, z_{4}=-2^{m-2}+1$, $z_{5}=2^{m-1}+\delta-1, z_{6}=2^{m-2}, z_{7}=2^{m-2}+1, z_{8}=1$ and $z_{9}=2^{m-2}+\delta+2$, where $\delta=0$ or $\delta=2^{m-1}$. If $m \geq 5$ then $2^{m-2} \in\left\langle 2^{3}\right\rangle$ and by Fig. 1 , it is easy to see that

$$
\begin{aligned}
E(Y)= & \left\{\mathcal{U}_{i} \mathcal{A}_{i}, \mathcal{U}_{i} \mathcal{B}_{i+2}, \mathcal{U}_{i} \mathcal{C}_{i+1}, \mathcal{U}_{i} \mathcal{D}_{i+1}, \mathcal{V}_{i} \mathcal{A}_{i}, \mathcal{V}_{i} \mathcal{B}_{i}, \mathcal{V}_{i} \mathcal{C}_{i+7}, \mathcal{V}_{i} \mathcal{D}_{i+1},\right. \\
& \left.\mathcal{W}_{i} \mathcal{A}_{i}, \mathcal{W}_{i} \mathcal{B}_{i+2}, \mathcal{W}_{i} \mathcal{C}_{i}, \mathcal{W}_{i} \mathcal{D}_{i+2}, \mathcal{X}_{i} \mathcal{A}_{i}, \mathcal{X}_{i} \mathcal{B}_{i}, \mathcal{X}_{i} \mathcal{C}_{i}, \mathcal{X}_{i} \mathcal{D}_{i} \mid i \in \mathbb{Z}_{8}\right\} .
\end{aligned}
$$


If $m=4$ then $2^{m-2}=4$ and similarly, one has

$$
\begin{aligned}
E(Y)= & \left\{\mathcal{U}_{i} \mathcal{A}_{i}, \mathcal{U}_{i} \mathcal{B}_{i+2}, \mathcal{U}_{i} \mathcal{C}_{i+5}, \mathcal{U}_{i} \mathcal{D}_{i+5}, \mathcal{V}_{i} \mathcal{A}_{i}, \mathcal{V}_{i} \mathcal{B}_{i}, \mathcal{V}_{i} \mathcal{C}_{i+7}, \mathcal{V}_{i} \mathcal{D}_{i+1},\right. \\
& \left.\mathcal{W}_{i} \mathcal{A}_{i}, \mathcal{W}_{i} \mathcal{B}_{i+6}, \mathcal{W}_{i} \mathcal{C}_{i}, \mathcal{W}_{i} \mathcal{D}_{i+6}, \mathcal{X}_{i} \mathcal{A}_{i}, \mathcal{X}_{i} \mathcal{B}_{i+4}, \mathcal{X}_{i} \mathcal{C}_{i+4}, \mathcal{X}_{i} \mathcal{D}_{i} \mid i \in \mathbb{Z}_{8}\right\}
\end{aligned}
$$

Thus, $Y$ is a $T$-reduced $\mathbb{Z}_{8}$-covering of $K_{4,4}$ with the voltage assignment $z_{1}=2$, $z_{2}=2, z_{3}=0, z_{4}=1, z_{5}=7, z_{6}=0, z_{7}=1, z_{8}=1, z_{9}=2$ when $m \geq 5$ and $z_{1}=2, z_{2}=6, z_{3}=4, z_{4}=5, z_{5}=7, z_{6}=4, z_{7}=5, z_{8}=1, z_{9}=6$ when $m=4$. Denote by $p_{2}$ the projection corresponding to this regular covering $Y$ of $K_{4,4}$. Since $\left\langle 2^{3}\right\rangle \leq K, \widetilde{X}$ is a $\left\langle 2^{3}\right\rangle$-covering of $Y$ with the covering transformation $\left\langle 2^{3}\right\rangle \cong \mathbb{Z}_{2^{m-3}}$ and denote by $p_{3}$ the projection corresponding to this regular covering $\widetilde{X}$ of $Y$. By definition of $Y$, it is clear that $p_{1}=p_{3} p_{2}$. Since $\widetilde{X}$ and $Y$ both have the same valency 4 and $\left\langle 2^{3}\right\rangle \triangleleft A$ is transitive on each fibre under $p_{3},\left\langle 2^{3}\right\rangle$ is the kernel of $A$ acting on $V(Y)$. Thus, $A /\left\langle 2^{3}\right\rangle$ is a subgroup of $\operatorname{Aut}(Y)$. Clearly, $A$ is fibre-preserving along the projection $p_{3}$. To prove that $A$ is fibre-preserving along $p_{1}$, it suffices to show that $\operatorname{Aut}(Y)$ is fibre-preserving along $p_{2}$ because $A /\left\langle 2^{3}\right\rangle \leq \operatorname{Aut}(Y)$ and $p_{1}=p_{3} p_{2}$.

By Tables 1 and $2, \bar{\alpha}_{1}, \bar{\beta}_{1}$ and $\bar{\beta}_{2}$ can be extended to the automorphisms of $\mathbb{Z}_{8}$ induced by $1 \mapsto 1,1 \mapsto-1$ and $1 \mapsto 1$ respectively when $m \geq 5$, and by $1 \mapsto 5$, $1 \mapsto-1$ and $1 \mapsto 5$ respectively when $m=4$. By Proposition 3.1, $\alpha_{1}, \beta_{1}$ and $\beta_{2}$ lift to automorphisms of $Y$ along the projection $p_{2}$. One may easily show that $\left|\left\langle\alpha_{1}, \beta_{1}, \beta_{2}\right\rangle\right|=2^{5}$ and so the lift of $\left\langle\alpha_{1}, \beta_{1}, \beta_{2}\right\rangle$ has order $2^{8}$. With the help of computer software package MAGMA [3], Aut $(Y)$ has order $2^{8}$ (also this can be obtained from the fact that the stabilizer of any given $\operatorname{arc}$ in $\operatorname{Aut}(Y)$ is a trivial group, which can be proved by considering the girth cycles of $Y$ together with arc-transitivity of the lift of $\left.\left\langle\alpha_{1}, \beta_{1}, \beta_{2}\right\rangle\right)$. It follows that the lift of $\left\langle\alpha_{1}, \beta_{1}, \beta_{2}\right\rangle$ is exactly the full automorphism group $\operatorname{Aut}(Y)$ of $Y$ because both of them have the same order. Thus, $\operatorname{Aut}(Y)$ is fibre-preserving along $p_{2}$ and so $A$ is fibre-preserving along $p_{1}$, as claimed.

Since $\left|A /\left\langle 2^{3}\right\rangle\right|=2^{m+5} / 2^{m-3}=2^{8}$, one has $\operatorname{Aut}(Y)=A /\left\langle 2^{3}\right\rangle$ because $A /\left\langle 2^{3}\right\rangle$ is a subgroup of $\operatorname{Aut}(Y)$ and $|\operatorname{Aut}(Y)|=2^{8}$, that is, the projection of $A$ along $p_{3}$ is $\operatorname{Aut}(Y)$. Note that $\operatorname{Aut}(Y)$ is fibre-preserving along $p_{2}$ and the projection of $\operatorname{Aut}(Y)$ along $p_{2}$ is $\left\langle\alpha_{1}, \beta_{1}, \beta_{2}\right\rangle$. Since $A$ is fibre-preserving along $p_{1}=p_{3} p_{2}$, the projection of $A$ along $p_{1}$ is also $\left\langle\alpha_{1}, \beta_{1}, \beta_{2}\right\rangle$. It follows that $\beta_{2}$ lifts along $p_{1}$, contrary to the fact that $\beta_{2}$ does not lift. Thus, $\operatorname{Aut}(\tilde{X})=H=B$ and so $\tilde{X}$ is $\frac{1}{2}$-arc-transitive.

To finish the proof, it remains to show that $\mathcal{C K}\left(2^{m}, i, 2^{m-2}+1\right)$ is of even radius and tightly attached for $i=1,2$. Note that $K_{4,4}$ is tightly $G_{1}$-attached and has two adjacent $G_{1}$-alternating cycles $(\mathbf{a}, \mathbf{u}, \mathbf{b}, \mathbf{v})$ and $(\mathbf{a}, \mathbf{w}, \mathbf{b}, \mathbf{x})$. Since the full automorphism group of $\mathcal{C K}\left(2^{m}, i, 2^{m-2}+1\right)$ is exactly the lift of $G_{1}$ for $i=1,2$, one may easily obtain two adjacent alternating cycles of $\mathcal{C K}\left(2^{m}, i, 2^{m-2}+1\right)$ having vertex sets $\left\{\mathbf{a}_{2 j}, \mathbf{b}_{2 j}, \mathbf{u}_{2 j}, \mathbf{v}_{2 j} \mid 2 j \in \mathbb{Z}_{2^{m}}\right\}$ and $\left\{\mathbf{a}_{2 j}, \mathbf{b}_{2 j}, \mathbf{w}_{2 j}, \mathbf{x}_{2 j} \mid 2 j \in \mathbb{Z}_{2^{m}}\right\}$. Thus, $\mathcal{C K}\left(2^{m}, i, 2^{m-2}+1\right)$ has radius $2^{m}$ and is tightly attached for $i=1,2$.

Remark The smallest ones in the two infinite families of half-arc-transitive graphs $\mathcal{C K}\left(2^{m}, i\right)$ for $i=1,2$ given in Lemma 5.2 are $\mathcal{C K}(16,1)$ and $\mathcal{C K}(16,2)$, which have 128 vertices. We depict $\mathcal{C} \mathcal{K}(16,1)$ in Fig. 2. By Lemma 4.1 and the definition of the graphs $\mathcal{C K}\left(2^{m}, i\right)$ for $i=1,2$ in the paragraph preceding Theorem 2.1, one has $\mathcal{C K}(16,1)=\mathcal{C K}(16,1,3)$ and hence it suffices to depict the graph $\mathcal{C K}(16,1,3)(\mathrm{de}-$ fined before Theorem 2.1). The four cycles with dotted lines are four alternating 
cycles with vertex sets

$$
\begin{array}{ll}
\left\{\mathbf{a}_{2 j}, \mathbf{u}_{2 j}, \mathbf{b}_{2 j}, \mathbf{v}_{2 j} \mid 2 j \in \mathbb{Z}_{16}\right\}, & \left\{\mathbf{a}_{2 j+1}, \mathbf{u}_{2 j+1}, \mathbf{b}_{2 j+1}, \mathbf{v}_{2 j+1} \mid 2 j \in \mathbb{Z}_{16}\right\}, \\
\left\{\mathbf{c}_{2 j}, \mathbf{w}_{2 j}, \mathbf{d}_{2 j}, \mathbf{x}_{2 j} \mid 2 j \in \mathbb{Z}_{16}\right\}, & \left\{\mathbf{c}_{2 j+1}, \mathbf{w}_{2 j+1}, \mathbf{d}_{2 j+1}, \mathbf{x}_{2 j+1} \mid 2 j \in \mathbb{Z}_{16}\right\} .
\end{array}
$$

The other four alternating cycles have vertex sets

$$
\begin{array}{ll}
\left\{\mathbf{a}_{2 j}, \mathbf{w}_{2 j}, \mathbf{b}_{2 j}, \mathbf{x}_{2 j} \mid 2 j \in \mathbb{Z}_{16}\right\}, & \left\{\mathbf{a}_{2 j+1}, \mathbf{w}_{2 j+1}, \mathbf{b}_{2 j+1}, \mathbf{x}_{2 j+1} \mid 2 j \in \mathbb{Z}_{16}\right\}, \\
\left\{\mathbf{c}_{2 j+1}, \mathbf{u}_{2 j}, \mathbf{d}_{2 j+1}, \mathbf{v}_{2 j} \mid 2 j \in \mathbb{Z}_{16}\right\}, & \left\{\mathbf{c}_{2 j}, \mathbf{u}_{2 j+1}, \mathbf{d}_{2 j}, \mathbf{v}_{2 j+1} \mid 2 j \in \mathbb{Z}_{16}\right\} .
\end{array}
$$

Clearly, if two adjacent vertices in an alternating cycle are labeled, then all vertices in the alternating cycle can be uniquely labeled. Thus, for each alternating cycle we only label two adjacent vertices in Fig. 2.

\section{Lemma 5.3 If $G_{2}$ lifts then $\widetilde{X}$ is not $\frac{1}{2}$-arc-transitive.}

Proof Suppose to the contrary that $\tilde{X}$ is $\frac{1}{2}$-arc-transitive. Since $G_{2}=\left\langle\alpha_{1}, \beta_{2}\right\rangle$ lifts, $\alpha_{1}^{*}$ and $\beta_{2}^{*}$ exist. Thus, Eqs. $1-8$ hold and so $\mathbb{Z}_{p^{m}}$ is generated by $z_{2}$ and $z_{8}$. Since $\beta_{2}^{*}$ exists, we have $1^{\beta_{2}^{*}}=t$ for some $t \in \mathbb{Z}_{p^{m}}^{*}$. By Table 2 , we have $t z_{1}=z_{2}-z_{3}$, $t z_{2}=z_{1}-z_{3}, t z_{3}=-z_{3}, t z_{4}=z_{9}-z_{7}, t z_{5}=-z_{7}, t z_{6}=z_{8}-z_{7}, t z_{7}=-z_{5}, t z_{8}=$ $z_{6}-z_{5}$ and $t z_{9}=z_{4}-z_{5}$. Since $\left(\beta_{2}^{*}\right)^{2}=1$, one has $t^{2}=1$. Substituting Eqs. 4 and 6 to $t z_{5}=-z_{7}$, one has $z_{2}=\left(k t^{-1}+k^{-3}\right) z_{8}=\left(k t+k^{-3}\right) z_{8}$ where $t^{-1}$ and $k^{-3}$ are the inverses of $t$ and $k^{3}$ in $\mathbb{Z}_{p^{m}}^{*}$ respectively. Thus, $\mathbb{Z}_{p^{m}}$ can be generated by $z_{8}$ and by Proposition 3.2, one may assume that $z_{8}=1$ and $z_{2}=k t+k^{-3}$. If $\beta_{1}$ lifts then the arc-transitive subgroup $\left\langle\alpha_{1}, \beta_{1}, \beta_{2}\right\rangle$ of $K_{4,4}$ lifts, contradicting half-arc-transitivity of $\widetilde{X}$. Thus, $\bar{\beta}_{1}$ cannot be extended to an automorphism of $\mathbb{Z}_{p^{m}}$ by Proposition 3.1.

Assume that $p$ is an odd prime. Then $t^{2}=1$ implies that $t=1$ or -1 because $\mathbb{Z}_{p^{m}}^{*}$ is cyclic. Let $t=1$. Then $t z_{3}=-z_{3}$ means that $z_{3}=0$. Since $z_{8}=1$, Eq. 2 implies $k=1$. Thus, $z_{2}=2$. By Eqs. $1-7, z_{1}=z_{9}=2, z_{3}=z_{6}=0, z_{5}=-1$ and $z_{4}=z_{7}=1$. By Table $1, \bar{\beta}_{1}$ can be extended to the automorphism of $\mathbb{Z}_{p^{m}}$ induced by $1 \mapsto-1$, a contradiction. Let $t=-1$. Then $t z_{6}=z_{8}-z_{7}$ means that $z_{6}+z_{8}=z_{7}$ and substituting Eqs. 5 and 6 to $z_{6}+z_{8}=z_{7}$, one has $(k-1)\left(k^{3}+1\right)=0$ because $z_{2}=$ $-k+k^{-3}$ and $z_{8}=1$. Thus, $k=1$ or $k^{3}=-1$ because the oddness of $p$ implies that $\left(k-1, p^{m}\right)=1$ or $\left(k^{3}+1, p^{m}\right)=1$. If $k=1$ then $z_{2}=0$ and $z_{8}=1$. By Eqs. $1-7$, $z_{1}=z_{3}=z_{6}=z_{9}=0$ and $z_{4}=z_{5}=z_{7}=1$. By Table $1, \bar{\beta}_{1}$ can be extended to the identity automorphism of $\mathbb{Z}_{p^{m}}$, a contradiction. If $k^{3}=-1$ then $\left(k-1, p^{m}\right)=1$ and by Eq. $8, z_{2}=0$. Since $z_{2}=-k+k^{-3}$, one has $k^{4}=1$ and so $k=-1$ because $k^{3}=$ -1 . Thus, $z_{2}=0$ and $z_{8}=1$. By Eqs. $1-7, z_{1}=z_{3}=z_{6}=-2, z_{4}=z_{5}=z_{7}=-1$ and $z_{9}=0$. By Table $1, \bar{\beta}_{1}$ can be extended to the automorphism of $\mathbb{Z}_{p^{m}}$ induced by $1 \mapsto-1$, a contradiction.

Assume that $p=2$. Then $k$ and $t$ are odd because $k, t \in \mathbb{Z}_{2^{m}}^{*}$. Since the smallest $\frac{1}{2}$-arc-transitive graph has order 27, one has $m \geq 2$ and so $t^{2}=1$ implies that $t=\delta+1$ or $\delta-1$, where $\delta=0$ or $2^{m-1}$. Suppose $t=\delta+1$. Then $z_{2}=k t+k^{-3}=k+k^{-3}+\delta$ because $k$ is odd. By Eq. $2, z_{3}$ is even and $t z_{3}=-z_{3}$ implies that $2 z_{3}=0$. It follows that $z_{3}=\delta_{1}$ where $\delta_{1}=0$ or $2^{m-1}$. Again by Eq. $2, z_{8}=1$ implies that $k=\delta_{1}+1$. Thus, $z_{2}=\delta+2$ and $z_{8}=1$. By Eqs. $1-7, z_{1}=\delta+\delta_{1}+2, z_{3}=\delta_{1}, z_{4}=\delta_{1}+1$, $z_{5}=\delta+\delta_{1}-1, z_{6}=\delta_{1}, z_{7}=\delta_{1}+1$ and $z_{9}=\delta+2$. By Table $1, \bar{\beta}_{1}$ can be extended to 
the automorphism of $\mathbb{Z}_{2^{m}}$ induced by $1 \mapsto \delta+\delta_{1}-1$, a contradiction. Thus, $t=\delta-1$. Then $z_{2}=k t+k^{-3}=-k+k^{-3}+\delta$, implying that $z_{2}$ is even. By Eq. $1, z_{1}$ is even. Since $t z_{1}=z_{2}-z_{3}$, one has $z_{1}+z_{2}=z_{3}$. Substituting Eqs. 1 and 2 to $z_{1}+z_{2}=z_{3}$, $\left(k^{2}-1\right)\left(k^{3}+k+1\right)=0$ because $z_{2}=-k+k^{-3}+\delta$ and $z_{8}=1$. Since $k^{3}+k+1$ is odd, $k^{2}=1$. Thus, $z_{2}=\delta$ and $z_{8}=1$. By Eqs. $1-7, z_{1}=k+\delta-1, z_{3}=k-1$, $z_{4}=k, z_{5}=k+\delta, z_{6}=k-1, z_{7}=k$ and $z_{9}=\delta$. By Table $1, \bar{\beta}_{1}$ can be extended to the automorphism of $\mathbb{Z}_{2^{m}}$ induced by $1 \mapsto k+\delta$, a contradiction. Thus, $\widetilde{X}$ cannot be $\frac{1}{2}$-arc-transitive when $G_{2}$ lifts.

Lemma 5.4 If $G_{3}$ lifts then $\widetilde{X}$ is not $\frac{1}{2}$-arc-transitive.

Proof Suppose to the contrary that $\tilde{X}$ is $\frac{1}{2}$-arc-transitive. Since $G_{3}=\left\langle\alpha_{2}, \beta_{1}\right\rangle$ lifts, $\alpha_{2}^{*}$ and $\beta_{1}^{*}$ exist. Let $1^{\alpha_{2}^{*}}=\ell$ for some $\ell \in \mathbb{Z}_{p^{m}}^{*}$. By Table $2, \ell z_{2}=z_{2}^{\alpha_{2}^{*}}=z_{8}-z_{7}$, that is, $z_{7}=-\ell z_{2}+z_{8}$. Similarly, by considering the images of $z_{6}, z_{8}, z_{7}, z_{1}$ and $z_{5}$ respectively, one may obtain that $z_{6}, z_{3}, z_{4}, z_{5}$ and $z_{9}$ are combinations of $z_{1}, z_{2}$ and $z_{8}$. Thus, we have the following equations

$$
\begin{aligned}
& z_{3}=z_{1}+\ell z_{2}+(\ell-1) z_{8} \\
& z_{4}=\ell^{-1} z_{1}-\left(\ell^{-1}+\ell^{2}+\ell\right) z_{2}+z_{8} \\
& z_{5}=\left(\ell^{-1}-\ell\right) z_{1}-\left(\ell^{-1}+\ell^{2}\right) z_{2}+z_{8} \\
& z_{6}=\ell^{-1} z_{1}-\ell^{-1} z_{2} \\
& z_{7}=-\ell z_{2}+z_{8} \\
& z_{9}=\left(\ell^{2}-1\right) z_{1}+\left(\ell^{3}-\ell+1\right) z_{2}+(1-\ell) z_{8} .
\end{aligned}
$$

which implies that $\mathbb{Z}_{p^{m}}$ can be generated by $z_{1}, z_{2}$ and $z_{8}$. Since $\left(\alpha_{2}^{*}\right)^{4}=1$, one has $\ell^{4}=1$.

Recall that $1^{\beta_{1}^{*}}=s$ for $s \in \mathbb{Z}_{p^{m}}$ and $s^{2}=1$. By Table $1, s z_{1}=-z_{1}, s z_{2}=z_{3}-z_{1}$, $s z_{3}=z_{2}-z_{1}, s z_{4}=z_{5}-z_{6}, s z_{5}=z_{4}-z_{6}, s z_{6}=-z_{6}, s z_{7}=z_{8}-z_{9}, s z_{8}=z_{7}-z_{9}$ and $s z_{9}=-z_{9}$. By Table $2, \ell z_{4}=z_{4}$ and since $\ell^{4}=1$, one has $\ell^{3}=\ell^{-1}$. Substituting Eqs. 9-14 to the equations $\ell z_{4}=z_{4}, s z_{2}=z_{3}-z_{1}, s z_{4}=z_{5}-z_{6}$ and $s z_{8}=z_{7}-z_{9}$, one may obtain

$$
\begin{aligned}
& \left(\ell^{3}-1\right) z_{1}+(1-\ell) z_{2}+(1-\ell) z_{8}=0 \\
& (s-\ell) z_{2}=(\ell-1) z_{8} \\
& \left(s \ell^{3}+\ell\right) z_{1}+\left(\ell^{2}-s \ell^{3}-s \ell^{2}-s \ell\right) z_{2}=(1-s) z_{8} \\
& \left(\ell^{2}-1\right) z_{1}+\left(\ell^{3}+1\right) z_{2}=(\ell-s) z_{8} .
\end{aligned}
$$

Based on Eqs. 9-18, we shall determine the voltages $z_{1}, z_{2}, \ldots, z_{9}$, which correspond to four columns for odd prime $p$ and three columns for $p=2$ in Table 3, where $t^{2}=-1$ and $\delta_{1}, \delta_{2}=0$ or $2^{m-1}$. For each column of values of $z_{1}, \ldots, z_{9}$, it will be shown that one of $\beta_{2}, \gamma_{1}$ and $\gamma_{3}$ lifts. Thus, $\left\langle\alpha_{2}, \beta_{1}, \gamma_{1}\right\rangle,\left\langle\alpha_{2}, \beta_{1}, \gamma_{3}\right\rangle$ or $\left\langle\alpha_{2}, \beta_{1}, \beta_{2}\right\rangle$ lifts and since these groups are arc-transitive on $K_{4,4}, \tilde{X}$ is arc-transitive. This is impossible because $\widetilde{X}$ is $\frac{1}{2}$-arc-transitive and hence the proof will be completed. 
Table 3 Voltages and corresponding lifts

\begin{tabular}{|c|c|c|c|c|c|c|c|}
\hline$p$ & odd & odd & odd & odd & 2 & 2 & 2 \\
\hline$z_{1}$ & 0 & $-1-t$ & 1 & 1 & $\delta_{1}$ & 1 & 1 \\
\hline$z_{2}$ & 0 & $-t$ & 0 & 1 & $\delta_{1}+\delta_{2}$ & $\delta_{1}$ & $\delta_{1}+1$ \\
\hline$z_{3}$ & 0 & -1 & 1 & 0 & $\delta_{2}$ & $\delta_{1}+1$ & $\delta_{1}$ \\
\hline$z .4$ & 1 & 0 & 1 & 0 & $\delta_{2}+1$ & $\delta_{1}+\delta_{2}+1$ & $\delta_{1}+\delta_{2}$ \\
\hline$z_{5}$ & 1 & $t$ & 0 & 0 & 1 & $\delta_{2}$ & $\delta_{2}$ \\
\hline$z_{6}$ & 0 & $t$ & 1 & 0 & $\delta_{2}$ & $\delta_{1}+1$ & $\delta_{1}$ \\
\hline$z_{7}$ & 1 & 0 & 0 & 1 & $\delta_{1}+\delta_{2}+1$ & $\delta_{1}+\delta_{2}$ & $\delta_{1}+\delta_{2}+1$ \\
\hline$z_{8}$ & 1 & 1 & 0 & 0 & 1 & $\delta_{2}$ & $\delta_{2}$ \\
\hline$z_{9}$ & 0 & 1 & 0 & 1 & $\delta_{1}+\delta_{2}$ & $\delta_{1}$ & $\delta_{1}+1$ \\
\hline Lift & $\beta_{2}$ & $\gamma_{1}$ & $\gamma_{3}$ & $\gamma_{3}$ & $\gamma_{3}$ & $\gamma_{3}$ & $\gamma_{3}$ \\
\hline
\end{tabular}

First assume that $p$ is odd. Then $s^{2}=1$ implies that $s=1$ or -1 . Let $s=1$. Then $s z_{1}=-z_{1}$ and $s z_{6}=-z_{6}$ imply that $z_{1}=z_{6}=0$, and by Eq. $12, z_{2}=0$. Thus, $\mathbb{Z}_{p^{m}}$ can be generated by $z_{8}$ and by Proposition 3.1, one may assume $z_{8}=1$. By Eq. 15 , $\ell=1$ and by Eqs. $9-14, z_{3}=z_{6}=z_{9}=0$ and $z_{4}=z_{5}=z_{7}=1$. By Table $2, \bar{\beta}_{2}$ can be extended to the automorphism of $\mathbb{Z}_{p^{3}}$ induced by $1 \mapsto-1$, which corresponds to column 2 of Table 3. Let $s=-1$. As $\ell^{4}=1$, the oddness of $p$ implies that $\ell^{2}=1$ or $\ell^{2}=-1$. If $\ell^{2}=-1$ then $(1+\ell)(1-\ell)=2$. Since $2 \in \mathbb{Z}_{p^{m}}^{*}$, we have $1+\ell, 1-\ell \in$ $\mathbb{Z}_{p^{m}}^{*}$. Furthermore, $(1+\ell)^{-1}=2^{-1}(1-\ell)$. By Eq. $16, z_{2}=2^{-1}(1-\ell)^{2} z_{8}=-\ell z_{8}$ and Eq. 15 implies that $z_{1}=-2 \ell(1+\ell)^{-1} z_{8}=-(1+\ell) z_{8}$ because $\ell^{2}=-1$. It follows that $\mathbb{Z}_{p^{m}}$ can be generated by $z_{8}$ and one may assume $z_{8}=1$. Thus, $z_{1}=$ $-1-\ell$ and $z_{2}=-\ell$. By Eqs. 9-14, $z_{3}=-1, z_{4}=0, z_{5}=\ell, z_{6}=\ell, z_{7}=0$ and $z_{9}=1$. By Table $1, \bar{\gamma}_{1}$ can be extended to the automorphism of $\mathbb{Z}_{p^{m}}$ induced by $1 \mapsto \ell$, which corresponds to column 3 of Table 3 . If $\ell^{2}=1$ then Eq. 17 implies $(\ell+1) z_{2}=z_{8}$ because $2 \in \mathbb{Z}_{p^{m}}^{*}$ and $s=-1$, and by Eq. 16 , one has $\ell z_{8}=0$, that is, $z_{8}=0$. Clearly, $\ell^{2}=1$ implies that $\ell=1$ or -1 . If $\ell=1$ then $z_{2}=0$ by Eq. 16 and if $\ell=-1$ then $z_{1}=z_{2}$ by Eq. 15 . Since $\mathbb{Z}_{p^{m}}$ is generated by $z_{1}, z_{2}$ and $z_{8}$, by Proposition 3.2, one may assume that $z_{1}=1$ for $\ell=1$ and $z_{1}=z_{2}=1$ for $\ell=-1$. By Eqs. 9-14, if $\ell=1$ then $z_{1}=z_{3}=z_{4}=z_{6}=1$ and $z_{2}=z_{5}=z_{7}=z_{8}=z_{9}=0$, and if $\ell=-1$ then $z_{1}=z_{2}=z_{7}=z_{9}=1$ and $z_{3}=z_{4}=z_{5}=z_{6}=z_{8}=0$. In these two cases, $\bar{\gamma}_{3}$ can be extended to the automorphisms of $\mathbb{Z}_{p^{m}}$ induced by $1 \mapsto-1$ and $1 \mapsto 1$ respectively, which correspond to columns 4 and 5 of Table 3 .

Now assume that $p=2$. Then $\mathbb{Z}_{2^{m}}^{*}$ consists of odd numbers in $\mathbb{Z}_{2^{m}}$. One may further assume $m \geq 2$ because the smallest $\frac{1}{2}$-arc-transitive graph has order 27 and so $s^{2}=1$ implies that $s=\delta+1$ or $\delta-1$, where $\delta=0$ or $2^{m-1}$. Note that $s= \pm 1$ when $m=2$. Thus, we have two cases, namely, case I: $s=\delta+1$ where $\delta=0$ or $2^{m-1}$ when $m \geq 3$ and $\delta=0$ when $m=2$, and case II: $s=\delta-1$ where $\delta=0$ or $2^{m-1}$ when $m \geq 3$ and $\delta=0$ when $m=2$.

Case I: $s=\delta+1$ where $\delta=0$ or $2^{m-1}$ when $m \geq 3$ and $\delta=0$ when $m=2$.

In this case, $\delta / 2+1$ is odd, that is, $\delta / 2+1 \in \mathbb{Z}_{2^{m}}^{*}$. Since $s z_{1}=-z_{1}$, one has $2(\delta / 2+1) z_{1}=0$ and so $2 z_{1}=0$. Similarly, $2 z_{6}=0$. It follows that $z_{1}, z_{6}=0$ or 
$2^{m-1}$. For convenience, write $z_{1}=\delta_{1}$ and $z_{6}=\delta_{2}$, where $\delta_{1}, \delta_{2}=0$ or $2^{m-1}$. By Eq. $12, z_{2}=z_{1}-\ell z_{6}=\delta_{1}+\delta_{2}$ because $\ell$ is odd. Thus, $\mathbb{Z}_{p^{m}}$ can be generated by $z_{8}$ because $z_{1}$ and $z_{2}$ are even, and by Proposition 3.2, one may assume that $z_{8}=1$. Since $\ell^{3}-1$ and $1-\ell$ are even, one has $\left(\ell^{3}-1\right) z_{1}=(1-\ell) z_{2}=0$ and by Eq. 15 , $\ell=1$. Since $z_{1}=\delta_{1}, z_{2}=\delta_{1}+\delta_{2}$ and $z_{8}=1$, by Eqs. 9-14, one has $z_{3}=\delta_{2}, z_{4}=$ $\delta_{2}+1, z_{5}=1, z_{6}=\delta_{2}, z_{7}=\delta_{1}+\delta_{2}+1$ and $z_{9}=\delta_{1}+\delta_{2}$. By Table $2, \bar{\gamma}_{3}$ can be extended to the automorphism of $\mathbb{Z}_{2^{m}}$ induced by $1 \mapsto-1$, which corresponds to column 6 of Table 3 .

Case II: $s=\delta-1$ where $\delta=0$ or $2^{m-1}$ when $m \geq 3$ and $\delta=0$ when $m=2$.

First we claim $\delta=0$. Clearly, one may assume that $m \geq 3$. Suppose to the contrary that $\delta=2^{m-1}$. Since $\ell^{4}=1$, one has $\ell= \pm 1,2^{m-1} \pm 1,2^{m-2} \pm 1$ or $2^{m-2}+2^{m-1} \pm 1$. It follows that $\ell^{2}=\sigma+1$, where $\sigma=0$ or $2^{m-1}$ when $m \geq 4$ and $\sigma=0$ when $m=3$. Clearly, $\ell^{3}=\ell+\sigma$ because $\ell \in \mathbb{Z}_{2^{m}}^{*}$ is odd. Since $s=\delta-1, s z_{1}=-z_{1}$ and $s z_{6}=-z_{6}$, one has $\delta z_{1}=0$ and $\delta z_{6}=0$. Then $z_{1}$ and $z_{6}$ are even because $\delta=2^{m-1}$. By Eq. 12, $z_{2}$ is even. Substituting $\ell^{2}=\sigma+1$ and $s=\delta-1$ to Eq. 17, one has $2 z_{8}=(\delta+\sigma) z_{1}+(2 \ell+2+\sigma+\delta) z_{2}+\delta z_{8}$, of which the right-hand side is a multiple of 4 because $m \geq 3$. This implies that if $z_{8}$ is odd then $2 \in\langle 4\rangle$ that is impossible. Thus, $z_{8}$ is even. It follows that $\mathbb{Z}_{2^{m}}$ is generated by three even elements $z_{1}, z_{2}$ and $z 8$, a contradiction. Thus, $\delta=0$, as claimed.

We now have $s=-1$ and $m \geq 2$. Note that each element in $\mathbb{Z}_{2^{m}}^{*}$ has order 2 or 1 when $m=2$ or 3 because $\mathbb{Z}_{2^{m}}^{*} \cong \mathbb{Z}_{2} \times \mathbb{Z}_{2^{m-2}}$. Then $\ell^{4}=1$ implies that $\ell^{2}=\sigma+1$, where $\sigma=0$ or $2^{m-1}$ when $m \geq 4$ and $\sigma=0$ when $m=2$ or 3 . Since $\ell$ is odd it follows that $\ell^{3}=\sigma+\ell$ and by Eq. 17, one has

$$
2 z_{8}=\sigma z_{1}+(2 \ell+2+\sigma) z_{2}
$$

The right-hand side of Eq. 19 is a multiple of 4, implying that $z_{8}$ is even. Since $\mathbb{Z}_{2^{m}}$ cannot be generated by three even elements, $z_{1}$ or $z_{2}$ is odd. Substituting $(1-\ell) z_{8}=$ $(1+\ell) z_{2}$ (obtained by Eq. 16 and $s=-1$ ) to Eq. 15 , one has

$$
2 z_{2}=\left(1-\ell^{3}\right) z_{1}=(1+\sigma-\ell) z_{1} .
$$

If $z_{1}$ is even then the right-hand side of Eq. 20 is a multiple of 4, implying that $z_{2}$ is even, a contradiction. Thus, $z_{1}$ is odd and so $z_{1}$ generates $\mathbb{Z}_{2^{m}}$. By Proposition 3.2, one may assume that $z_{1}=1$.

We now claim that $\sigma=0$. Since $(1+\ell) z_{2}=(1-\ell) z_{8}$ (Eq. 16), by Eq. 18 one has $(\ell+1) z_{8}=\sigma z_{1}+(\sigma+\ell+1) z_{2}=\sigma z_{1}+\sigma z_{2}+(\ell+1) z_{2}=\sigma z_{1}+\sigma z_{2}+(1-\ell) z_{8}$. It follows that $2 z_{8}=\sigma z_{1}+\sigma z_{2}$ because $\ell$ is odd, and by Eq. 19, 2( $\left.\ell+1\right) z_{2}=0$. Thus, Eq. 20 implies that $0=(\ell+1)(1+\sigma-\ell)=1-\ell^{2}=\sigma$ because $z_{1}=1$.

So far we have proved that $s=-1$ and $\ell^{2}=1$. Since $z_{1}=1$ and $\sigma=0$, by Eqs. 19 and 20 one has $2 z_{2}=1-\ell$ and $2 z_{8}=(\ell+1)(1-\ell)=0$. Since $(1+\ell) z_{2}=(1-\ell) z_{8}$, one has $(1+\ell) z_{2}=0$ because $2 z_{8}=0$ and $1-\ell$ is even. Note that $\ell^{2}=1$ implies that $\ell= \pm 1$ or $2^{m-1} \pm 1$ because $m \geq 2$. Using $2 z_{2}=1-\ell$ and $(1+\ell) z_{2}=0$, one may easily show that $\ell \neq 2^{m-1} \pm 1$. It follows that $\ell=1$ or -1 . If $\ell=1$ then $2 z_{2}=2 z_{8}=0$ and if $\ell=-1$ then $2\left(z_{2}-1\right)=2 z_{8}=0$. Thus, one may assume that $z_{2}=\delta_{1}$ and $z_{8}=\delta_{2}$ for $\ell=1$, and $z_{2}=1+\delta_{1}$ and $z_{8}=\delta_{2}$ for $\ell=-1$, where $\delta_{1}, \delta_{2}=0$ or $2^{m-1}$. Recall that $z_{1}=1$. By Eqs. 9-14, one has if $\ell=1$ then $z_{3}=\delta_{1}+1$, 
$z_{4}=\delta_{1}+\delta_{2}+1, z_{5}=\delta_{2}, z_{6}=\delta_{1}+1, z_{7}=\delta_{1}+\delta_{2}$ and $z_{9}=\delta_{1}$, and if $\ell=-1$ then $z_{3}=\delta_{1}, z_{4}=\delta_{1}+\delta_{2}, z_{5}=\delta_{2}, z_{6}=\delta_{1}, z_{7}=\delta_{1}+\delta_{2}+1$ and $z_{9}=\delta_{1}+1$. By Table 2 , $\bar{\gamma}_{3}$ can be extended to the automorphisms of $\mathbb{Z}_{2^{m}}$ induced by $1 \mapsto-1$ for $\ell=1$ and by $1 \mapsto 1$ for $\ell=-1$, which correspond to columns 7 and 8 of Table 3 .

Acknowledgements This work was supported by the National Natural Science Foundation of China (10571013), the Key Project of Chinese Ministry of Education (106029), the Specialized Research Fund for the Doctoral Program of Higher Education in China (20060004026), and Com² MaC-KOSEF (R111999-054) in Korea. We would like to thank the referees for valuable comments and suggestions.

\section{References}

1. Alspach, B., \& Xu, M. Y. (1992). 1/2-transitive graphs of order 3p. J. Algebr. Comb., 1, $275-282$.

2. Alspach, B., Marušič, D., \& Nowitz, L. (1994). Constructing graphs which are 1/2-transitive. J. Aust. Math. Soc. A, 56, 391-402.

3. Bosma, W., Cannon, C., \& Playoust, C. (1997). The MAGMA algebra system I The user language. J. Symb. Comput., 24, 235-265.

4. Bouwer, I. Z. (1970). Vertex and edge-transitive but not 1-transitive graphs. Canad. Math. Bull., 13, 231-237.

5. Conder, M. D. E., \& Marušič, D. (2003). A tetravalent half-arc-transitive graph with non-abelian vertex stabilizer. J. Comb. Theory B, 88, 67-76.

6. D’Azevedo, A. B., \& Nedela, R. (2004). Half-arc-transitive graphs and chiral hypermaps. Eur. J. Comb., 25, 423-436.

7. Djoković, D. Z. (1974). Automorphisms of graphs and coverings. J. Comb. Theory B, 16, 243-247.

8. Du, S. F., \& Xu, M. Y. (1999). Vertex-primitive $\frac{1}{2}$-arc-transitive graphs of smallest order. Comm. Algebra, 27, 163-171.

9. Fang, X. G., Li, C. H., \& Xu, M. Y. (2004). On edge-transitive Cayley graphs of valency four. Eur. J. Comb., 25, 1107-1116.

10. Feng, Y.-Q., \& Kwak, J. H. (2004). s-Regular cubic graphs as coverings of the complete bipartite graph $K_{3,3}$. J. Graph Theory, 45, 101-112.

11. Feng, Y.-Q., \& Wang, K. S. (2003). s-Regular cubic graphs as coverings of the three dimensional hypercube $Q_{3}$. Eur. J. Comb., 24, 719-731.

12. Feng, Y.-Q., Kwak, J. H., \& Wang, K. S. (2005). Classifying cubic symmetric graphs of order $8 p$ or $8 p^{2}$. Eur. J. Comb., 26, 1033-1052.

13. Feng, Y.-Q., Wang, K. S., \& Zhou, C. X. (2007). Tetravalent half-transitive graphs of order $4 p$. Eur. J. Comb., 28, 726-733.

14. Gross, J. L., \& Tucker, T. W. (1977). Generating all graph coverings by permutation voltage assignment. Discrete Math., 18, 273-283.

15. Holt, D. F. (1981). A graph which is edge transitive but not arc transitive. J. Graph Theory, 5, 201204.

16. Hong, S., Kwak, J. H., \& Lee, J. (1996). Regular graph coverings whose covering transformation groups have the isomorphism extension property. Discrete Math., 168, 85-105.

17. Li, C. H., \& Sim, H. S. (2001). On half-transitive metacirculant graphs of prime-power order. J. Comb. Theory $B, 81,45-57$.

18. Li, C. H., Lu, Z. P., \& Marušič, D. (2004). On primitive permutation groups with small suborbits and their orbital graphs. J. Algebra, 279, 749-770.

19. Li, C. H., Lu, Z. P., \& Zhang, H. (2006). Tetravalent edge-transitive graphs with odd number of vertices. J. Comb. Theory B, 96, 164-181.

20. Malnič, A. (1998). Group actions, coverings and lifts of automorphisms. Discrete Math., 182, 203218.

21. Malnič, A., \& Marušič, D. (1993). Imprimitive graphs and graph coverings. In D. Jungnickel, S. A. Vanstone (Eds.). Coding theory, design theory, group theory, Proceedings of M. Hall memorial conference (pp. 221-229). New York: Wiley

22. Malnič, A., \& Marušič, D. (1999). Constructing 4-valent $\frac{1}{2}$-transitive graphs with a nonsolvable automorphism group. J. Comb. Theory B, 75, 46-55. 
23. Malnič, A., \& Marušič, D. (2002). Constructing $\frac{1}{2}$-arc-transitive graphs of valency 4 and vertex stabilizer $\mathbb{Z}_{2} \times \mathbb{Z}_{2}$. Discrete Math., 245, 203-216.

24. Malnič, A., \& Potočnik, P. (2006). Invariant subspaces, duality, and covers of the Petersen graph. Eur. J. Comb., 27, 971-989.

25. Malnič, A., Marušič, D., \& Potočnik, P. (2004). On cubic graphs admitting an edge-transitive solvable group. J. Algebr. Comb., 20, 99-113.

26. Malnič, A., Marušič, D., \& Potočnik, P. (2004). Elementary abelian covers of graphs. J. Algebr. Comb., 20, 71-97.

27. Malnič, A., Marušič, D., Potočnik, P., \& Wang, C. Q. (2004). An infinite family of cubic edge- but not vertex-transitive graphs. Discrete Math., 280, 133-148.

28. Malnič, A., Nedela, R., \& Škoviera, M. (2000). Lifting graph automorphisms by voltage assignments. Eur. J. Comb., 21, 927-947.

29. Marušič, D. (1998). Half-transitive group actions on finite graphs of valency 4. J. Comb. Theory B, 73, 41-76.

30. Marušič, D. (1998). Recent developments in half-transitive graphs. Discrete Math., 182, 219-231.

31. Marušič, D. (2005). Quartic half-arc-transitive graphs with large vertex stabilizers. Discrete Math., 299, 180-193.

32. Marušič, D., \& Nedela, R. (1998). Maps and half-transitive graphs of valency 4. Eur. J. Comb., 19, 345-354.

33. Marušič, D., \& Nedela, R. (2001). Partial line graph operator and half-arc-transitive group actions. Math. Slovaca, 51, 241-257.

34. Marušič, D., \& Nedela, R. (2001). On the point stabilizers of transitive groups with non-self-paired suborbits of length 2. J. Group Theory, 4, 19-43.

35. Marušič, D., \& Nedela, R. (2002). Finite graphs of valency 4 and girth 4 admitting half-transitive group actions. J. Aust. Math. Soc., 73, 155-170.

36. Marušič, D., \& Pisanski, T. (1999). Weakly flag-transitive configurations and $\frac{1}{2}$-transitive graphs. Eur. J. Comb., 20, 559-570.

37. Marušič, D., \& Praeger, C. E. (1999). Tetravalent graphs admitting half-transitive group actions alternating cycles. J. Comb. Theory B, 75, 185-205.

38. Marušič, D., \& Waller, A. (2000). Half-transitive graphs of valency 4 with prescribed attachment numbers. J. Graph Theory, 34, 89-99.

39. Marušič, D., \& Xu, M. Y. (1997). A $\frac{1}{2}$-transitive graph of valency 4 with a nonsolvable group of automorphisms. J. Graph Theory, 25, 133-138.

40. Šajna, M. (1998). Half-transitivity of some metacirculants. Discrete Math., 185, 117-136.

41. Škoviera, M. (1986). A contribution to the theory of voltage graphs. Discrete Math., 61, 281-292.

42. Taylor, D. E., \& Xu, M. Y. (1994). Vertex-primitive $\frac{1}{2}$-transitive graphs. J. Aust. Math. Soc. Ser. A, 57, 113-124.

43. Tutte, W. T. (1966). Connectivity in graphs. Toronto: University of Toronto Press.

44. Wang, R. J. (1994). Half-transitive graphs of order a product of two distinct primes. Commun. Algebra, 22, 915-927.

45. Wilson, S. (2004). Semi-transitive graphs. J. Graph Theory, 45, 1-27.

46. Xu, M. Y. (1992). Half-transitive graphs of prime-cube order. J. Algebr. Comb., 1, 275-282.

47. Zhou, C. X., \& Feng, Y.-Q. (2006). An infinite family of tetravalent half-arc-transitive graphs. Discrete Math., 306, 2205-2211. 